\title{
1.52-GHz Micromechanical Extensional Wine-Glass Mode Ring Resonators
}

\author{
Yuan Xie, Member, IEEE, Sheng-Shian Li, Member, IEEE, Yu-Wei Lin, Student Member, IEEE, \\ Zeying Ren, Member, IEEE, and Clark T.-C. Nguyen, Fellow, IEEE
}

\begin{abstract}
Vibrating polysilicon micromechanical ring resonators, using a unique extensional wine-glass-mode shape to achieve lower impedance than previous UHF resonators, have been demonstrated at frequencies as high as $1.2 \mathrm{GHz}$ with a $Q$ of 3,700 , and $1.52 \mathrm{GHz}$ with a $Q$ of 2,800 . The 1.2-GHz resonator exhibits a measured motional resistance of $1 \mathrm{M} \Omega$ with a dc-bias voltage of $20 \mathrm{~V}$, which is 2.2 times lower than the resistance measured on radial contourmode disk counterparts at the same frequency. The use of larger rings offers a path toward even lower impedance, provided the spurious modes that become more troublesome as ring size increases can be properly suppressed using methods described herein. With spurious modes suppressed, the high- $Q$ and low-impedance advantages, together with the multiple frequency on-chip integration advantages afforded by capacitively transduced $\mu$ mechanical resonators, make this device an attractive candidate for use in the front-end RF filtering and frequency generation functions needed by wireless communication devices.
\end{abstract}

\section{INTRODUCTION}

$\mathrm{H}$ AVING recently been demonstrated at frequencies past $1 \mathrm{GHz}$, some with quality factors $(Q ' s)>10,000$ in both vacuum and air [1], [2], vibrating micromechanical (" $\mu$ mechanical") resonators $[3]-[6]$ are emerging as possible enablers for on-chip versions of the pre-select (or even channel-select) filters needed in the RF front-ends of wireless communication devices. Such filters are designed much like the macroscopic mechanical filters of old [7], where high- $Q$ mechanical resonators are mechanically coupled together into networks that realize desired frequency shaping transmission responses. So far, the frequencies of properly designed (i.e., properly terminated) versions of such filters have reached only the very high frequency (VHF) [8]-[10] range, although unterminated responses showing coupledresonator mode peaks in the ultra-high frequency (UHF) range have been demonstrated [11]-[13]. The availability of properly terminated on-chip RF filters in the UHF range

Manuscript received February 22, 2007; accepted December 10, 2007. This work was supported by DARPA Grant No. F30602-01-10573 .

The authors were with the Department of Electrical Engineering and Computer Science, University of Michigan, Ann Arbor, MI (email: yxie@umich.edu).

Y. Xie is now with Avago Technologies, San Jose, CA.

S.-S. Li is now with RF Micro Devices, Greensboro, NC

Y.-W. Lin is now with Broadcom Corporation, Irvine, CA.

Z. Ren and C. T.-C. Nguyen are now with the Department of Electrical Engineering and Computer Sciences, University of California, Berkeley, CA.

Digital Object Identifier 10.1109/TUFFC.2008.725 and beyond could greatly simplify the realization of future multi-band reconfigurable wireless communicators, which are expected to require many more RF filters than today's communicators - e.g., one set for each supported communication standard, as shown in Fig. 1(a). Because the simplicity of capacitively transduced resonators has so far enabled them to achieve the highest $Q$ 's and most design flexibility (i.e., reconfigurability) among micromechanical resonators to date, capacitively transduced resonators are of high interest for front-end RF filtering strategies, especially in schemes where channel-selection right at $\mathrm{RF}$ is desired [14].

Unfortunately, however, although their $Q$ 's and frequencies are now sufficient, the greater than $1 \mathrm{M} \Omega$ impedances of the $\mathrm{GHz}$ range capacitively transduced $\mu$ mechanical resonators demonstrated so far are still too high to allow matching to conventional RF stages and components, which today are often designed to match to a front-end system impedance of $50 \Omega$. The use of $50 \Omega$ is a convention that derives mainly from the need to route signals through relatively high capacitance environments, such as those of the pc boards generally used for electronic system integration. However, as more components are integrated onto a single silicon chip, e.g., using the technology of the present work, system impedances need no longer adhere to a $50 \Omega$ convention because off-chip boardlevel capacitors need no longer be driven. As a result, system impedances will likely rise to take advantage of certain noise benefits. For example, the use of a high system impedance helps to desensitize a system from losses arising from parasitic resistance (e.g., wire resistance). It further allows more optimal noise matching to transistor-based functions, for which noise figure can be minimized when driven by optimal source resistances, which are generally higher than $50 \Omega$. However, even when completely integrated on-chip, system impedances will likely still not rise past the kilohm range because finite chip-level capacitance will still place a limit on the magnitude of impedance. Thus, design methodologies that allow reduction and tailoring of capacitive-transducer impedances down to the kilohm range are still desirable.

As a potential solution to this need, this paper presents a ring-resonator structure shown in Fig. 1(b) that operates in a compound $(2,2)$ mode capable of achieving higher frequency and lower impedance than previous stand-alone (as opposed to arrayed [15]) UHF micromechanical resonators. This resonant-mode shape, shown in Fig. 2(a), combines aspects of two previously demonstrated modes, namely, 

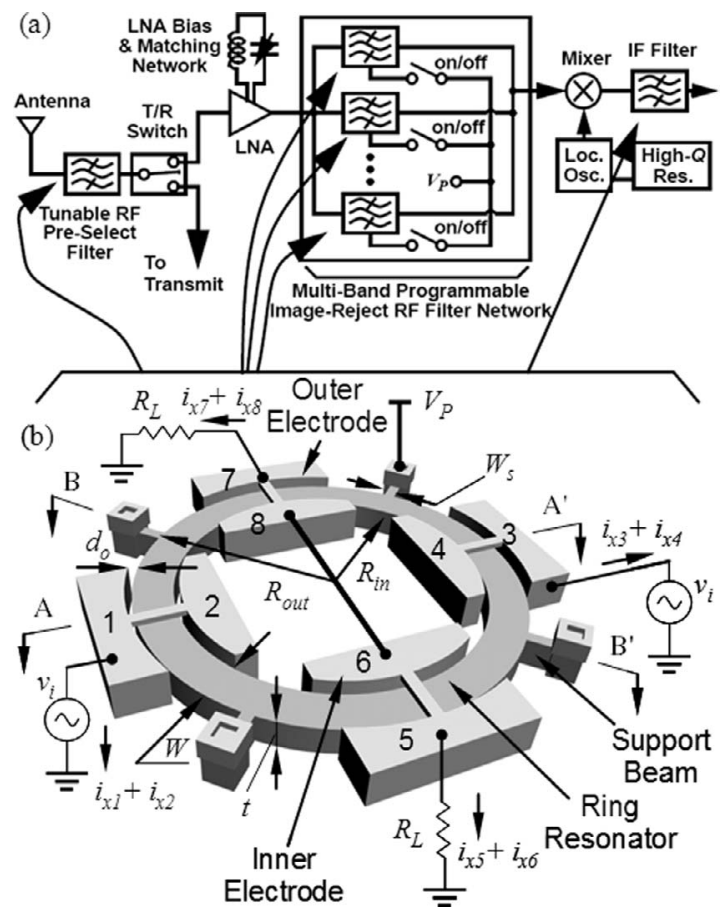

Fig. 1. (a) System block diagram of a multi-band reconfigurable wireless front-end, indicating targeted RF and IF filtering components; (b) perspective-view schematic of an extensional wine-glass ring resonator, identifying key features and a typical drive and sense configuration.


Fig. 2. Comparison of ANSYS-simulated resonance vibration mode shapes for (a) an extensional wine-glass mode ring resonator; and (b) a radial-contour mode solid-disk resonator.

the extensional radial-contour vibration mode [3] and the compound (2,1)-mode (i.e., the mode often dubbed the "wine-glass disk" mode in previous papers [16], [17]), together with the geometric advantages of a ring structure [18], to achieve the best of each design. In particular, this compound $(2,2)$ mode design, dubbed in [19] the "extensional wine-glass ring," or "EWGR," allows: 1) a high resonance frequency, owing to its use of an extensional mode; 2) a lower motional impedance than a filled disk, due to its ring-geometry, which offers a larger capacitive transducer overlap area than provided by the perimeter of a filled disk; 3 ) potentially higher $Q$, since its mode shape resembles a wine-glass-like mode [16], which allows its support structure to avoid a centrally located stem and thereby reduce anchor losses, as seen in [16]; and 4) a mode shape conducive to fully balanced differential inputs and outputs. With this design, frequencies as high as $1.2-\mathrm{GHz}$ with $Q$ 's around 3,700 , and at $1.52-\mathrm{GHz}$ with a $Q$ of 2,800 , have been demonstrated, with motional resistances 2.2 times lower than resistances measured on radial contour-mode disk counterparts [3].

The paper begins with a description of the basic structure and operation of the device in Section II, then develops the analytical formulations needed to attain a specified frequency and equivalent circuit model in Section III. After verifying the accuracy of the analytical formulations with the fabrication and measurements of Sections IV and $\mathrm{V}$, Section VI then addresses the issue of spurious modes, proposing and experimentally assessing various methods by which they can be eliminated.

\section{Device Structure And Operation}

The key to attaining the high $Q$ of this resonator is in the support flexibility afforded by its extensional wineglass-mode shape, shown in Fig. 2(a). In this mode shape, the expansion (contraction) of the two diagonal quarters of the ring exhibit extensional characteristics, while the deformed inner and outer perimeters of the ring display wineglass mode features. In contrast to the purely radial mode of a solid disk resonator, shown in Fig. 2(b), where the only nodes are at the disk center or (for higher modes) at less accessible circles within the disk area, this extensional wine-glass mode offers several quasinodal points away from the center, at perimeter locations where the ring may be supported. As demonstrated by previous (lower frequency) solid-disk wine-glass resonators [16], which achieved $Q$ 's 145,000 at $61 \mathrm{MHz}[20]$, the ability to support a disk resonator at its perimeter rather than its center can also be advantageous for attaining high $Q$. In addition, perimeter supports allow a simpler fabrication process sequence than needed for stem-supported structures, which often require self-alignment of the stem for maximum $Q[1]$.

Fig. 1(b) presents the perspective-view schematic of the actual resonator design used in this work, identifying key features and an excitation configuration that instigates the extensional wine-glass mode of Fig. 2(a). As shown, this device consists of a ring suspended $650 \mathrm{~nm}$ above the substrate by four tethers connected at quasinodal locations for this mode, where there are no radial displacements, but still tangential ones. The support beams are designed with geometries that isolate the resonator structure from its anchors to minimize energy losses to the substrate, allowing the structure to retain its highest $Q$. Aside from the support structure, multiple electrodes surround the ring, both inside and outside, to maximize the transducer overlap capacitance. To excite the device, a dc-bias voltage $V_{P}$ is applied to the conductive ring and an ac voltage $v_{i}$ to drive electrodes along one radial axis of the ring. To- 
gether, these voltages generate incremental electrostatic radial forces at the frequency of $v_{i}$ at each drive electrode $j$ given by

$$
F_{d j}=\frac{1}{2}\left(V_{P j}-v_{i}\right)^{2} \frac{\partial C_{j}}{\partial r} \Rightarrow \delta F_{d j}=-V_{P j} \frac{\partial C_{j}}{\partial r} v_{i}
$$

that compress and expand the ring extensionally at their locations, driving the device into the resonance vibration mode shape of Fig. 2(a) when the drive frequency matches the resonance frequency $f_{o}$. In (1), $V_{P j}=V_{P}-V_{j}, V_{j}$ is the dc voltage applied to electrode $j$ (usually grounded), $C_{o j}$ is the electrode-to-ring overlap capacitance at port $j$, and $\partial C_{j} / \partial r$ is the change in this capacitance per unit radial displacement, which for the direction convention indicated in Fig. 5 (to be discussed later), has a positive value at the ring's outer edge, but a negative value at the ring's inner edge. To illustrate the sign conventions of (1): With $V_{P}$ a finite value, $V_{j}=0 \mathrm{~V}$, and $v_{i}$ going positive, (1) indicates that the incremental force $\delta F_{d j}$ acting on the outer ring edge is negative; i.e., it acts inward according to the convention of Fig. 5, where the positive radial direction points outward from the ring center. This effectively moves the outer edge inward, compressing the ring. Meanwhile, the incremental force predicted by (1) due to this same rising $v_{i}$ acting on the inner ring edge is positive, resulting in an outward movement of the inner ring edge, also compressing the ring.

Once vibrating, dc-biased (by $V_{P}$ ) time-varying electrode-to-resonator capacitors generate currents at each electrode $j$ given by

$$
i_{x j}=V_{P j} \frac{d C_{j}}{d t}=V_{P j} \frac{d C_{j}}{d r} \frac{d r}{d t},
$$

where the phase of $i_{x j}$ depends on the phase of $d C_{j} / d t$. In particular, as the ring vibrates, the shape of the extensional wine-glass mode is such that when the portions of the ring between the input drive electrodes-1 to 4 in Fig. 1(b)-compress, those between the output sense electrodes -5 to 8 in Fig. 1(b) - expand. At this instant, $d C_{j} / d t$ is negative at the drive electrodes, meaning that motional currents enter the input electrodes. At the same time, $d C_{j} / d t$ is positive at the sense electrodes, meaning that motional currents exit from the output electrodes. Thus, the net current into the ring structure itself is zero for the specific excitation configuration of Fig. 1(b). (Note that this is not necessarily the case for other configurations, and the difference will be described later.)

From a "black box" perspective, this device looks like any other electrical filter from its input and output. This despite the fact that its operation mechanism is largely mechanical, in that electrical input signals are first converted to mechanical signals, processed (with high $Q$ ) in the mechanical domain, then re-converted to electrical signals at the output, ready for further processing by subsequent transceiver stages. As will be shown in subsequent sections, with a finite $V_{P}$ this mechanical resonator device is electrically equivalent to an $L C R$ tank circuit, and so has a response identical to that for any resonator, except with very high $Q$.

In addition to providing analytical formulations for force and current, (1) and (2) also emphasize the on/off switchable feature of this device. In particular, when $V_{P}$ is finite, the device operates as described. But when $V_{P}=0 \mathrm{~V}$, the force at the frequency of $v_{i}$ goes to zero, so the device cannot be driven at the frequency of $v_{i}$. Closer inspection of the full force equation in the left side of (1) reveals that the device can still be driven to resonance by a much weaker force generated by $v_{i}$ inputs at half the resonance frequency. However, with $V_{P}=0 \mathrm{~V}$, no current is generated at the output electrodes, effectively rendering the device dormant, or "off." In effect, with $V_{P}=0 \mathrm{~V}$, the device is an open circuit.

\section{Device Design And Modeling}

Having discussed basic qualitative operational aspects of the EWGR, expressions governing the precise design of this device are now in order. These include formulations for resonance frequency, $Q$ maximization, and lumped parameter equivalent mechanical and electrical circuits. Each of these is now addressed.

\section{A. Resonance Frequency Design}

For the case where no voltages are applied (i.e., no dcbias $V_{P}$; purely mechanical resonance), the nominal resonance frequency $f_{o}$ for an EWGR is given by [21]

$$
f_{\text {nom }}=\frac{h}{2 \pi} \sqrt{\frac{E}{\rho\left(1-\sigma^{2}\right)}},
$$

where $\rho, \sigma$, and $E$ are the density, Poisson ratio, and Young's modulus, respectively, of the ring structural material, and $h$ is a parameter that satisfies

$$
\operatorname{det}\left[H_{i j}\right]_{4 \times 4}=0
$$

where the elements of the matrix $\left[H_{i j}\right]_{4 \times 4}$ can be expressed as (5) (see next page) where $R_{\text {inner }}$ and $R_{\text {outer }}$ are the inner and outer radius of the ring, respectively, shown in Fig. $1(\mathrm{~b}) ; h^{2} / k^{2}=(1-\sigma) / 2, J_{n}(x)$ and $Y_{n}(x)$ are Bessel functions of the first and second kind, respectively; and $n$ is the circumferential order of the mode shape $(n=2$ for the extensional wine-glass mode).

The resonance frequency of an EWGR is most accurately specified via simultaneous solution of above complicated set of expressions and matrices. But considering the extensional wine-glass mode is comprised largely of expansion and contraction about the ring width, which is similar to the longitudinal vibration of a bar, for intuitive purposes, the resonance frequency can be approximately specified by

$$
f_{\text {nom } \mid \text { approx. }}=\frac{m}{2 W} \sqrt{\frac{E}{\rho}}, m=1,3,5, \cdots,
$$




$$
\begin{aligned}
& H_{11}=\left[\left(k R_{\text {outer }}\right)^{2} / 2-n(n+1)\right] J_{n}\left(h R_{\text {outer }}\right)+h R_{\text {outer }} J_{n-1}\left(h R_{\text {outer }}\right) \\
& H_{12}=\left[\left(k R_{\text {outer }}\right)^{2} / 2-n(n+1)\right] Y_{n}\left(h R_{\text {outer }}\right)+h R_{\text {outer }} Y_{n-1}\left(h R_{\text {outer }}\right) \\
& H_{13}=n(n+1) J_{n}\left(k R_{\text {outer }}\right)-n k R_{\text {outer }} J_{n-1}\left(k R_{\text {outer }}\right) \\
& H_{14}=n(n+1) Y_{n}\left(k R_{\text {outer }}\right)-n k R_{\text {outer }} Y_{n-1}\left(k R_{\text {outer }}\right) \\
& H_{21}=\left[\left(k R_{\text {inner }}\right)^{2} / 2-n(n+1)\right] J_{n}\left(h R_{\text {inner }}\right)+h R_{\text {inner }} J_{n-1}\left(h R_{\text {inner }}\right) \\
& H_{22}=\left[\left(k R_{\text {inner }}\right)^{2} / 2-n(n+1)\right] Y_{n}\left(h R_{\text {inner }}\right)+h R_{\text {inner }} Y_{n-1}\left(h R_{\text {inner }}\right) \\
& H_{23}=n(n+1) J_{n}\left(k R_{\text {inner }}\right)-n k R_{\text {inner }} J_{n-1}\left(k R_{\text {inner }}\right) \\
& H_{24}=n(n+1) Y_{n}\left(k R_{\text {inner }}\right)-n k R_{\text {inner }} Y_{n-1}\left(k R_{\text {inner }}\right) \\
& H_{31}=n h R_{\text {outer }} J_{n-1}\left(h R_{\text {outer }}\right)-n(n+1) J_{n}\left(h R_{\text {outer }}\right) \\
& H_{32}=n h R_{\text {outer }} Y_{n-1}\left(h R_{\text {outer }}\right)-n(n+1) Y_{n}\left(h R_{\text {outer }}\right) \\
& H_{33}=-k R_{\text {outer }} J_{n-1}\left(k R_{\text {outer }}\right)+\left[n(n+1)-\left(k R_{\text {outer }}\right)^{2} / 2\right] J_{n}\left(k R_{\text {outer }}\right) \\
& H_{34}=-k R_{\text {outer }} Y_{n-1}\left(k R_{\text {outer }}\right)+\left[n(n+1)-\left(k R_{\text {outer }}\right)^{2} / 2\right] Y_{n}\left(k R_{\text {outer }}\right) \\
& H_{41}=n h R_{\text {inner }} J_{n-1}\left(h R_{\text {inner }}\right)-n(n+1) J_{n}\left(h R_{\text {inner }}\right) \\
& H_{42}=n h R_{\text {inner }} Y_{n-1}\left(h R_{\text {inner }}\right)-n(n+1) Y_{n}\left(h R_{\text {inner }}\right) \\
& H_{43}=-k R_{\text {inner }} J_{n-1}\left(k R_{\text {inner }}\right)+\left[n(n+1)-\left(k R_{\text {inner }}\right)^{2} / 2\right] J_{n}\left(k R_{\text {inner }}\right) \\
& H_{44}=-k R_{\text {inner }} Y_{n-1}\left(k R_{\text {inner }}\right)+\left[n(n+1)-\left(k R_{\text {inner }}\right)^{2} / 2\right] Y_{n}\left(k R_{\text {inner }}\right)
\end{aligned}
$$

where $W=\left(R_{\text {outer }}-R_{\text {inner }}\right)$ is the ring width, shown in Fig. 1(b), and $m$ is the order of the vibration mode.

To design the resonator with specified resonance frequency and ring outer radius $R_{\text {outer }}$, as well as material constants of the ring structure, (6) is first used to find the approximate inner ring radius $R_{\text {inner }}$, then simultaneous numerical solution of (3)-(5) is used to find the exact $R_{\text {inner }}$ using the value provided by (6) as an initial guess. For example, for a $433-\mathrm{MHz}$ resonator with an outer radius of $40 \mu \mathrm{m},(6)$ gives an inner radius of $30.7 \mu \mathrm{m}$ and (3) gives $h=3.28 \times 10^{5}[1 / \mathrm{m}]$. Plugging $h$ and $R_{\text {outer into }}(5)$ and using $R_{\text {inner }}=30.7 \mu \mathrm{m}$ as an initial guess, then solving (4) by iteration, yields the inner radius $R_{\text {inner }}=30.5 \mu \mathrm{m}$.

\section{B. Effect of Electrical Stiffness}

Although (3)-(5) correctly model the purely mechanical resonance behavior of the ring structure, they must be modified when electrical inputs are used to excite its resonance. In particular, when a dc-bias voltage $V_{P}$ is dropped across an electrode-to-resonator gap, the electric field variations caused by the oscillating structure during resonance give rise to electrically derived restoring forces in phase with the mechanical motion, hence, equivalent to a stiffness - an electrical stiffness $k_{e}$. The impact of $k_{e}$ can be modeled by a procedure similar to that of [8], where its effect on frequency can be expressed by

$$
\begin{aligned}
f_{o} & =\frac{1}{2 \pi} \sqrt{\frac{k_{r e}}{m_{r e}}}=\frac{1}{2 \pi} \sqrt{\frac{k_{m}-k_{e}}{m_{r e}}} \\
& =f_{\text {nom }}\left[1-\left\langle\frac{k_{e}}{k_{m}}\right\rangle\right]^{1 / 2} \text { and } \\
f_{\text {nom }} & =\frac{1}{2 \pi} \sqrt{\frac{k_{m}}{m_{r e}}}=\frac{h}{2 \pi} \sqrt{\frac{E}{\rho\left(1-\sigma^{2}\right)}},
\end{aligned}
$$

where $m_{r e}$ and $k_{r e}$ are the lumped equivalent mass and stiffness of the resonator, whose expressions are given in subsection $\mathrm{D}, k_{m}$ is the purely mechanical stiffness with no voltages applied to the resonator, and the quantity $\left\langle k_{e} / k_{m}\right\rangle$ must be obtained via integration over the angle $\theta$ of the electrodes surrounding the ring. As shown in (7), the electrical stiffnesses of all electrodes, regardless of their position (inside or outside the ring), subtract from the mechanical spring constant $k_{m}$ of the ring, reducing its resonance frequency.

The $\left\langle k_{e} / k_{m}\right\rangle$ term in (7) can be determined by integrating differential contributions of electrical stiffness [22]

$$
d k_{e j}=V_{P j}^{2} \frac{\varepsilon_{o} R_{r} t d \theta}{(d(\theta))^{3}}, R_{r}=R_{\text {inner }} \text { or } R_{\text {outer }}
$$

over each electrode-to-resonator overlap region, to yield

$$
\left\langle\frac{k_{e}}{k_{m}}\right\rangle=\sum_{j=1}^{8}\left[\int_{\theta_{j 1}}^{\theta_{j 2}} \frac{d k_{e j}}{k_{m}\left(R_{r}, \theta\right)}\right], R_{r}=R_{\text {inner }} \text { or } R_{\text {outer }}
$$

where $k_{m}\left(R_{r}, \theta\right)$ is the mechanical stiffness given in (14) and $\theta_{j 1}$ and $\theta_{j 2}$ are the start and end angles of the $j$ th electrode in Fig. 1(b).

For UHF resonators such as disks [3] and the rings in this work, $k_{m}$ is usually on the order of $10^{6}$ to $10^{8} \mathrm{~N} / \mathrm{m}$, while $k_{e}$ is only on the order of $10^{2}$ to $10^{3} \mathrm{~N} / \mathrm{m}$. Since the $k_{m}$ 's of ring resonators are orders of magnitude higher than their $k_{e}$ 's, typical fractional frequency changes due to electrical stiffness are only on the order of 1 to $100 \mathrm{ppm}$. In particular, for the $30.5 \mu \mathrm{m}$ inner radius, $50 \mu \mathrm{m}$ outer radius, $2-\mu \mathrm{m}$-thick 220-MHz EWGR of this work with $V_{P}=5 \mathrm{~V}$ and $d_{o}=53 \mathrm{~nm}, k_{m}=15.4 \mathrm{MN} / \mathrm{m}$ and $k_{e}=1206 \mathrm{~N} / \mathrm{m}$, and (7)-(9) predict a fractional frequency shift of only 


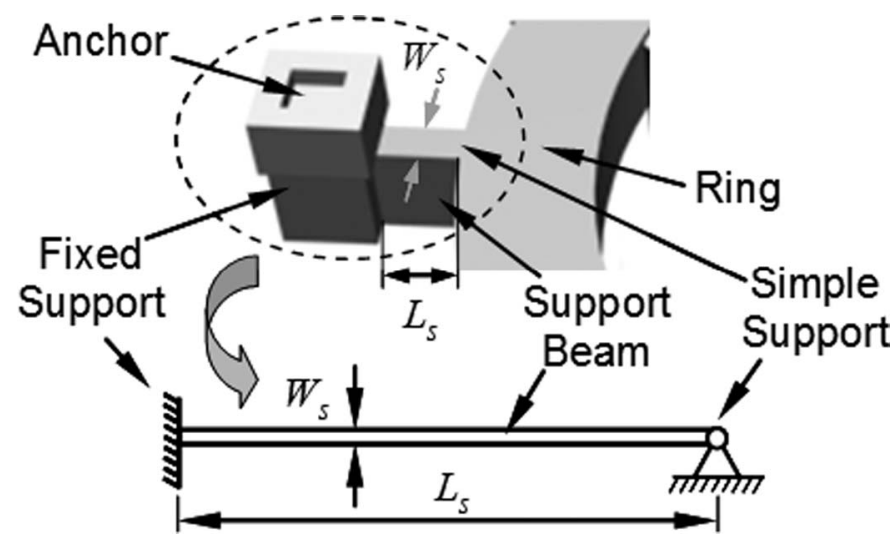

Fig. 3. Schematic of an EWGR support beam, equating it to a beam with simple-fixed boundary conditions.

$18.5 \mathrm{ppm}$, which is close to the measured $20.4 \mathrm{ppm}$ for this device, c.f., Fig. 16(a). For the 1.5-GHz device of Section $\mathrm{V}$, the difference factor between $k_{m}=214 \mathrm{MN} / \mathrm{m}$ and $k_{e}=630 \mathrm{~N} / \mathrm{m}$ is even larger, and the frequency shift is only $0.5 \mathrm{ppm}$, which is practically negligible.

\section{Support Beam Design}

In the present design, the support beams are sized to maximize the $Q$ of the compound $(2,2)$ extensional wineglass mode. In particular, although the support attachment points correspond to nodes in the radial direction, they are actually not rotational nodes, so in-plane rotations still occur at these points. To isolate this rotational motion from the anchors, the support beams are designed to vibrate in a simple-fixed in-plane flexural mode, illustrated schematically in Fig. 3, at the extensional wine-glass resonance frequency. Here, the fixed end condition at the anchor is obvious, and the simple end condition at the attachment point to the ring was verified via finite-element analysis to provide a good fit to the rotational behavior at that attachment point. This strategy is similar to the use of quarter-wavelength supports for previous free-free beam [23] and wine-glass disk [16] resonators, because it essentially implements a mechanical transformer that transforms the infinite mechanical impedance at the anchor to near zero impedance at the ring attachment points, allowing the ring to vibrate unimpeded, hence, with its maximum $Q$.

With these boundary conditions, the length of the support beam $L_{S}$ that achieves support resonance at frequency $f_{o}$ is given by [24]

$$
L_{s}=\sqrt{\frac{\lambda_{i}^{2} W_{s}}{4 \pi \sqrt{3} f_{o}} \sqrt{\frac{E}{\rho}}}, i=1,2, \cdots,
$$

where $W_{S}$ is the width of the support beam, and $\lambda_{i}$ satisfies

$$
\tan \lambda_{i}=\tanh \lambda_{i}
$$

An approximate solution to (11) is

$$
\lambda_{i}=(4 i+1) \frac{\pi}{4}, i=1,2, \cdots .
$$

Given the above formulations, the support beam for a $435-\mathrm{MHz}$ EWGR (such as that summarized in Table I of Section V) that yields maximum $Q$ should have a length of $4.6 \mu \mathrm{m}$ and a width of $1.6 \mu \mathrm{m}$. For the 1.5-GHz EWGR of Table I, the length and width dimensions should be $4.4 \mu \mathrm{m}$ and $1.6 \mu \mathrm{m}$ for maximum $Q$. The measurements of Section V will later quantify the degree to which (10)-(12) actually achieve maximum $Q$.

\section{Small-Signal Electrical Equivalent Circuit}

As with other vibrating resonators, the equivalent $L C R$ circuit for the EWGR is governed by the total integrated kinetic energy in the resonator, by its mode shape, and by parameters associated with its transducer ports [7]. Fig. 4(a) presents the complete equivalent circuit for the 8-port device of Fig. 1(b), where the core resonator is modeled by a series $L C R$ tank, and all eight of its ports are represented by transformers. Each top terminal of the eight ports corresponds to an electrode, while the bottom terminals are all tied to a common port representing the ring resonator itself. The values of the $L C R$ elements in the circuit of Fig. 4(a) depend directly on the mass $m_{r}$, stiffness $k_{r}$, and damping $c_{r}$, of the resonator, and are given by $[7]$

$$
l_{x}=m_{r e}, c_{x}=1 / k_{r e}, r_{x}=c_{r e},
$$

where $m_{r e}, k_{r e}$, and $c_{r e}$, are the lumped equivalent mass, stiffness, and damping, respectively, of the ring at locations where the displacement (or velocity) is maximum. (Expressions for all of these variables will be forthcoming.) For the device of Fig. 1(b), these locations are at the midpoints of the electrodes.

The equivalent mass $m_{r}(r, \theta)$ at a location $(r, \theta)$ can be obtained by dividing the total kinetic energy of the ring by one-half the square of the velocity at that location [7]. Doing so for the case of a location $\left(R_{\text {inner }}, \theta\right)$ or $\left(R_{\text {outer }}, \theta\right)$ on either the inner or outer perimeter of the ring yields the following expressions for the equivalent mass and stiffness:

$$
\begin{aligned}
& \rho t \int_{R_{\text {inner }}}^{R_{\text {outer }}} \int_{0}^{2 \pi}\left(\left[U_{r}(r, \theta)\right]^{2}+\left[U_{\theta}(r, \theta)\right]^{2}\right) r d r d \theta \\
& m_{r}\left(R_{r}, \theta\right) {\left[U_{r}\left(R_{r}, \theta\right)\right]^{2}+\left[U_{\theta}\left(R_{r}, \theta\right)\right]^{2} } \\
& k_{m}\left(R_{r}, \theta\right)=\omega_{\text {nom }}^{2} m_{r}\left(R_{r}, \theta\right), \text { and } \\
& k_{r}\left(R_{r}, \theta\right)=\omega_{o}^{2} m_{r}\left(R_{r}, \theta\right),
\end{aligned}
$$

where $\omega_{o}$ and $\omega_{\text {nom }}$ are the radian forms of $f_{o}$ and $f_{\text {nom }}$, respectively. In (14), $U_{r}(r, \theta)$ and $U_{\theta}(r, \theta)$ are the radial and tangential displacements of the ring, given by [21] 

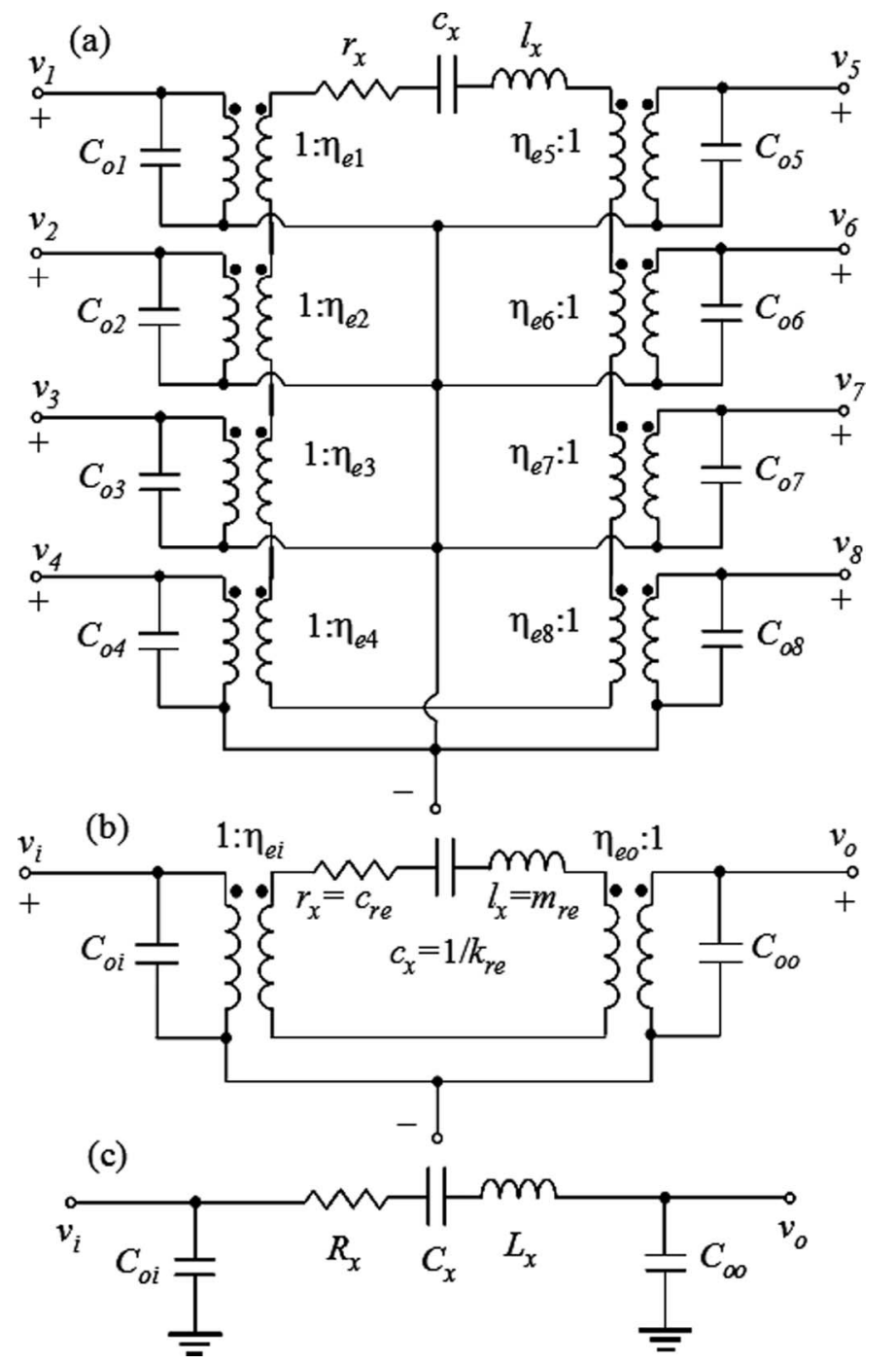

Fig. 4. (a) Complete equivalent circuit model for the EWGR, showing eight ports total (four inner and four outer electrodes); (b) physically consistent equivalent circuit model using actual values of mass, stiffness and damping for elements in a two-port configuration when all four input and output ports are connected together, respectively; and (c) transformer-less electrical circuit model obtained by reduction of (b).

$$
\begin{aligned}
U_{r}(r, \theta)= & \frac{1}{h} \cos (2 \theta)\left[\frac{d J_{n}(h r)}{d r}+\frac{B}{A} \frac{d Y_{n}(h r)}{d r}\right. \\
& \left.+\frac{2}{r} \cdot\left(\frac{C}{A} J_{n}(k r)+\frac{D}{A} Y_{n}(k r)\right)\right] \text { and } \\
U_{\theta}(r, \theta)= & -\frac{1}{h} \sin (2 \theta)\left[\frac{2}{r} \cdot\left(J_{n}(h r)+\frac{B}{A} Y_{n}(h r)\right)\right. \\
& \left.+\frac{C}{A} \frac{d J_{n}(k r)}{d r}+\frac{D}{A} \frac{d Y_{n}(k r)}{d r}\right]
\end{aligned}
$$

where constants $B / A, C / A$, and $D / A$ can be obtained by solving the expression

$$
\left[H_{i j}\right]_{4 \times 4}\left[\begin{array}{llll}
A & B & C & D
\end{array}\right]^{T}=0 .
$$

The equivalent damping at locations on the ring edges can then be expressed in terms of mass, stiffness, and $Q$, as follows:

$$
c_{r}\left(R_{r}, \theta\right)=\frac{\omega_{o} m_{r}\left(R_{r}, \theta\right)}{Q}=\frac{\sqrt{k_{r}\left(R_{r}, \theta\right) \cdot m_{r}\left(R_{r}, \theta\right)}}{Q} .
$$

When evaluated at $\theta=0$, which is a maximum velocity point, expressions for $m_{r e}, k_{r e}$, and $c_{r e}$ become

$$
\begin{aligned}
m_{r e} & =m_{r}\left(R_{r}, 0\right), k_{r e}=k_{r}\left(R_{r}, 0\right), \\
c_{r e} & =\frac{\sqrt{k_{r e} m_{r e}}}{Q}, \text { where } \\
R_{r} & =R_{\text {inner }} \text { or } R_{\text {outer }}
\end{aligned}
$$

The electromechanical coupling coefficient $\eta_{e j}$ characterizing the $j$ th port of the resonator corresponding to the $j$ th electrode in Fig. 1(b) can be determined using a procedure similar to that in [8] and [25]. Doing so yields

$$
\eta_{e j}=V_{P j} \cdot\left\langle\frac{\partial C_{j}}{\partial r}\right\rangle
$$

where

$$
\begin{aligned}
\left\langle\frac{\partial C_{j}}{\partial r}\right\rangle= & \left(\begin{array}{c}
\int_{\theta_{1}}^{\theta_{2}} \int_{\theta_{1}}^{\theta_{2}}\left\{\frac{2\left(\varepsilon_{o} t\right)^{2}}{\left[d(\theta) d\left(\theta^{\prime}\right)\right]^{2}} \cdot \frac{\cos 2 \theta}{\cos 2 \theta^{\prime}} \cdot k_{r}\left(R_{\text {inner }}, 0\right) \cdot\right. \\
\left.\left[\frac{R_{\text {inner }}^{2}}{k_{r}\left(R_{\text {inner }}, \theta^{\prime}\right)}+\frac{R_{\text {inner }} R_{\text {outer }} / b}{k_{r}\left(R_{\text {outer }}, \theta^{\prime}\right)}\right]\right\} d \theta d \theta^{\prime}
\end{array}\right)^{\frac{1}{2}} \\
& j=2,4,6 \text { and } 8
\end{aligned}
$$

and

$$
\begin{aligned}
\left\langle\frac{\partial C_{j}}{\partial r}\right\rangle= & \left(\begin{array}{c}
\int_{\theta_{1}}^{\theta_{2}} \int_{\theta_{1}}^{\theta_{2}}\left\{\frac{2\left(\varepsilon_{o} t\right)^{2}}{\left[d(\theta) d\left(\theta^{\prime}\right)\right]^{2}} \cdot \frac{\cos 2 \theta}{\cos 2 \theta^{\prime}} \cdot k_{r}\left(R_{\text {outer }}, 0\right) \cdot\right. \\
\left.\left[\frac{R_{\text {outer }}^{2}}{k_{r}\left(R_{\text {outer }}, \theta^{\prime}\right)}+\frac{b R_{\text {inner }} R_{\text {outer }}}{k_{r}\left(R_{\text {inner }}, \theta^{\prime}\right)}\right]\right\} d \theta d \theta^{\prime}
\end{array}\right)^{\frac{1}{2}} \\
& j=1,3,5 \text { and } 7 .
\end{aligned}
$$

In (20) and (21), $t$ is the thickness of the ring; $\varepsilon_{o}$ is the permittivity in vacuum; $\omega_{o}$ is the radian resonance frequency; $d(\theta)$ is the electrode-to-resonator gap spacing, which can vary with $\theta$ due to the imbalance of electrostatic forces generated by the dc-biases applied between the ring and its inner and outer electrodes; $Q$ is the quality factor; $V_{P j}=V_{P}-V_{j}$ is the dc-bias voltage across the resonatorto-electrode gap; $\theta_{1}$ and $\theta_{2}$ are the start and end angles of one pair of the inner and outer output electrodes in the polar coordinate system as indicated in Fig. 5 ; and $b$ is the ratio of displacement at the outer and inner ring perimeter at a given angle. The brackets around the $\left\langle\partial C_{j} / \partial r\right\rangle$ terms in (19)-(21) are there to emphasize that these are integrated quantities that include the dependence of displacement on the ring mode shape during resonance.

The $C_{o}$ 's in each circuit of Fig. 4 represent the static electrode-to-resonator overlap capacitance, which is on the order of $50 \mathrm{fF}$ for a typical quarter-circle electrode on a a $31.8-\mu \mathrm{m}$ inner radius, $40-\mu \mathrm{m}$ outer radius, $2-\mu \mathrm{m}$-thick 1.52-GHz EWGR. 


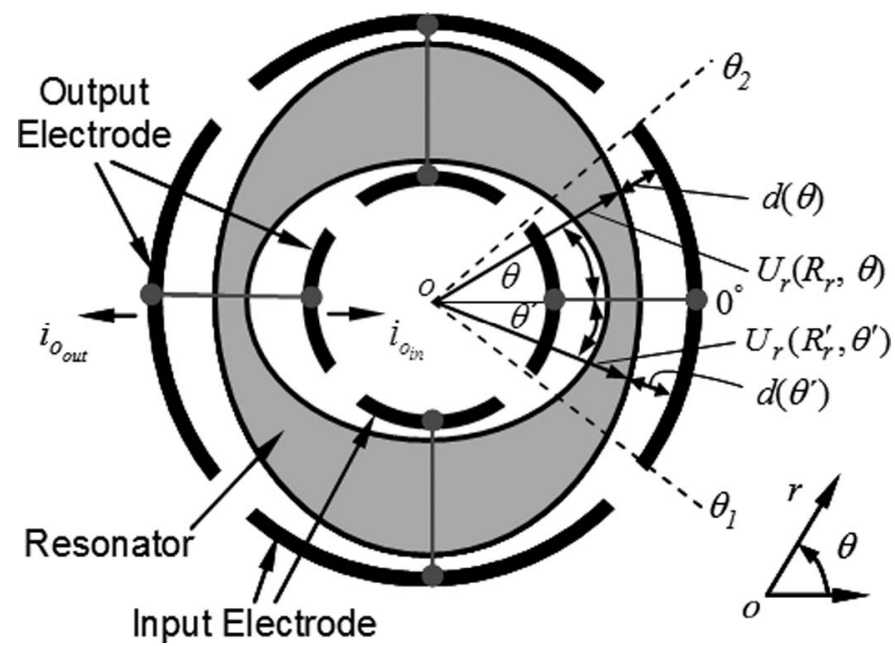

Fig. 5. Top-view schematic of the EWGR used to determine analytical expressions for electrical equivalent circuit elements. Here, the resonator itself, its eight electrodes, and variables used in (20) and (21), are all indicated; $U_{r}\left(R_{r}, \theta\right)$ is the radial displacement at the ring perimeter; and four horizontal electrodes are connected as input electrodes, and four vertical electrodes are connected as output electrodes.

When four of the eight electrodes are connected together as an input port (ports 1-4) and the other four connected as an output port (ports 5-8), respectively, as shown in the two-port configuration in Fig. 1(b), Fig. 4(a) reduces to the simplified two-port equivalent circuit shown in Fig. 4(b) and (c) with element values given by

$$
R_{x}=\frac{c_{r e}}{\eta_{e}^{2}}, C_{x}=\frac{\eta_{e}^{2}}{k_{r e}}, L_{x}=\frac{m_{r e}}{\eta_{e}^{2}}
$$

where $\eta_{e}$ is now the total electromechanical coupling coefficient of the composite input (or output) port. For the particular hookup of Fig. 1(b), where all inner ports are identically sized, and all outer ports likewise, $\eta_{e}$ is given by

$$
\eta_{e}=\sqrt{2 \eta_{e, \text { inner }}^{2}+2 \eta_{e, \text { outer }}^{2} \cdot \frac{m_{r}\left(R_{\text {inner }}, 0\right)}{m_{r}\left(R_{\text {outer }}, 0\right)}}
$$

when $c_{r e}$ in $(22)$ is taken at the inner perimeter, or

$$
\eta_{e}=\sqrt{2 \eta_{e, \text { inner }}^{2} \cdot \frac{m_{r}\left(R_{\text {outer }}, 0\right)}{m_{r}\left(R_{\text {inner }}, 0\right)}+2 \eta_{e, \text { outer }}^{2}}
$$

when $c_{r e}$ in (22) is taken at the outer perimeter. In (23) and $(24), \eta_{e, \text { inner }}$ and $\eta_{e, \text { outer }}$ are the electromechanical coupling factors for a single inner port and a single outer port, respectively, of Fig. 1(b), given by the expression for $\eta_{e j}$ in (19). The input and output shunt capacitors (neglecting the electrode-to-substrate capacitance which is usually much smaller than the electrode-to-resonator overlap capacitance) combine those of the individual electrodes to yield

$$
C_{o}=2\left(C_{\text {inner }}+C_{\text {outer }}\right)
$$

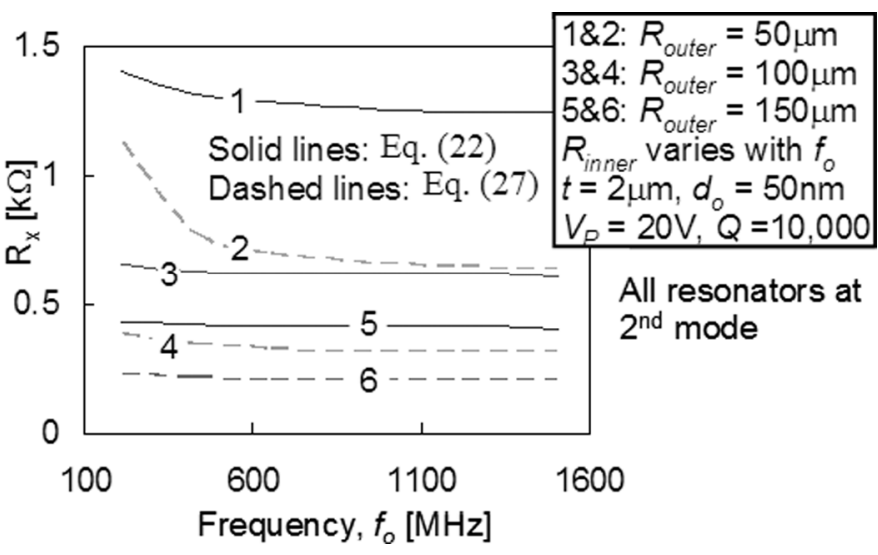

Fig. 6. Predicted curves of motional resistance versus (second mode) resonance frequency using (22) and (27) for EWGRs with different inner and outer radii.

where $C_{\text {inner }}$ and $C_{\text {outer }}$ are the single port inner and single port outer electrode-to-resonator shunt capacitors, respectively, given by

$$
\begin{aligned}
& C_{\text {inner }}=\varepsilon_{o} R_{\text {inner }}\left(\theta_{2}-\theta_{1}\right) t / d_{o}, \text { and } \\
& C_{\text {outer }}=\varepsilon_{o} R_{\text {outer }}\left(\theta_{2}-\theta_{1}\right) t / d_{o} .
\end{aligned}
$$

The above formulations, although very accurate (as will be verified in Section V), are quite complex and, thus, do not readily provide the design insights that a simpler formulation might allow. Pursuant to providing a more manageable expression that enables some design insight, the motional resistance $R_{x}$ in (22) can be simplified by neglecting the distributed mass and stiffness of the ring over the electrode overlap regions and assuming that all electrodes are at dc ground, which yields

$$
R_{x}=\frac{\omega_{o} m_{r e}}{Q V_{P}^{2}} \cdot \frac{d_{o}^{4}}{\varepsilon_{o}^{2} P_{o e}^{2} t^{2}},
$$

where $P_{o e}=2\left(R_{\text {outer }}+R_{\text {inner }}\right)\left(\theta_{2}-\theta_{1}\right)$ is the average overlap perimeter.

Fig. 6 shows the predicted curves of motional resistance versus (second mode) resonance frequency using (22) and (27) for a series of resonators with varying outer radii $R_{\text {outer }}$. As seen, the motional resistance calculated from (27) is smaller than that from (22), since the former neglects the actual distributed stiffness of the ring [8]. Values provided by (27), however, are useful as a rough estimate of the motional resistance, and they clearly mimic the trends predicted by (22), which verifies the utility of (27) for design insight. From (27), the series motional resistance of this device can be tailored by changing the adjustable parameters, such as the electrode-to-resonator gap spacing $d_{o}$, for which there is a very strong fourth order dependence; the dc-bias voltage $V_{P}$, for which there is a square law dependence; the thickness $t$, for which the dependence ends up being relatively linear, despite the square law indicated by (27), due to the equivalent mass $m_{r e}$ dependence on $t$; and finally, the average overlap perimeter $P_{o e}$ of the ring, for which the dependence is also more linear 
than the square law indicated in (27), again due to the implicit equivalent mass $m_{r e}$ dependence on $R_{\text {inner }}$ and $R_{\text {outer }}$ in (14). Fig. 7 shows plots of motional resistance versus several of the above parameters for a $900-\mathrm{MHz}$ resonator, indicating the design parameters needed to achieve $10 \Omega$ impedance, which would be needed to achieve a filter matched to $50 \Omega$ with decent insertion loss.

Due to its use of an extensional mode, the frequency of an EWGR is determined primarily by the width of its ring, and not by its radius. Thus, the perimeter of the device can be made arbitrarily large by increasing its average radius to maximize its transducer capacitance, hence, drive down its series motional resistance $R_{x}$. Section $\mathrm{V}$ will present actual measurements verifying this design strategy. In addition, since the frequency of this device is determined primarily by its lateral dimensions, which are set by CAD layout, this device easily supports multiple frequencies on a single chip without the need for multiple film depositions. In contrast, counterparts with frequencies determined only by thickness [e.g., thin-film bulk acoustic resonators (FBARs)] require an additional film deposition for each additional frequency. For future multi-band wireless communication devices that might require more than ten different RF filters to address multiple communication standards, filters realized using a resonator with thicknessdefined frequency might require ten different film depositions, at which point it might very likely be less expensive to implement each filter separately, rather than on a single-chip. In contrast, the lateral dimension-defined frequency of the EWGR device of this work allows realization of ten different frequencies with a single film deposition and frequencies defined by CAD layout, which are all very amenable to single-chip implementation, with obvious cost advantages.

\section{FABRICATION}

Extensional wine-glass resonators with frequencies ranging from $200 \mathrm{MHz}$ to more than $1.5 \mathrm{GHz}$ were designed and fabricated using the self-aligned stem process described in [3], which combines polysilicon surfacemicromachining with a sacrificial sidewall spacer technique to achieve phosphorus-doped polysilicon structures with polysilicon side electrodes, and with nano-scale electrodeto-resonator lateral gaps. Fig. 8 presents cross sections for a finished EWGR taken through the A-A' and B-B' lines indicated in Fig. 1(b). Since these rings are edge-supported devices, rather than center-supported (as are the disks of $[1],[3])$, the self-alignment feature of this process is actually not necessary. Rather, a simpler process, such as that of [26], or a polysilicon electrode derivative of it, should suffice. In this respect, EWGR devices arguably might have a cost advantage over center-stem-supported disks.

Fig. 9 presents scanning electron micrographs (and dimensions) for 435-MHz and 651-MHz EWGRs, as well as a zoom-in shot clearly showing the $85 \mathrm{~nm}$ lateral electrodeto-resonator gap achieved via the fabrication process. As
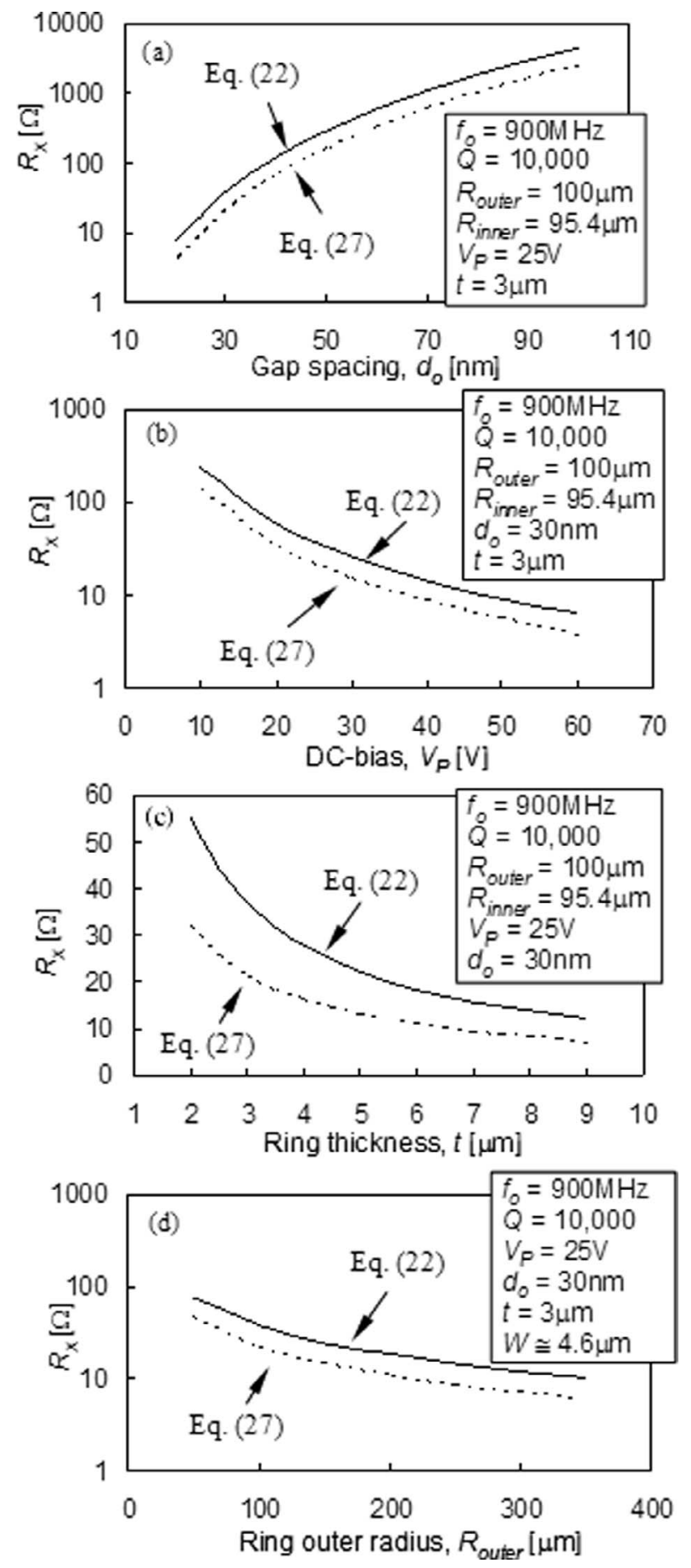

Fig. 7. Predicted curves of motional resistance versus (a) gap spacing; (b) dc-bias; (c) ring thickness; and (d) ring outer radius, showing methods by which an impedance of $10 \Omega$ might be achieved. 


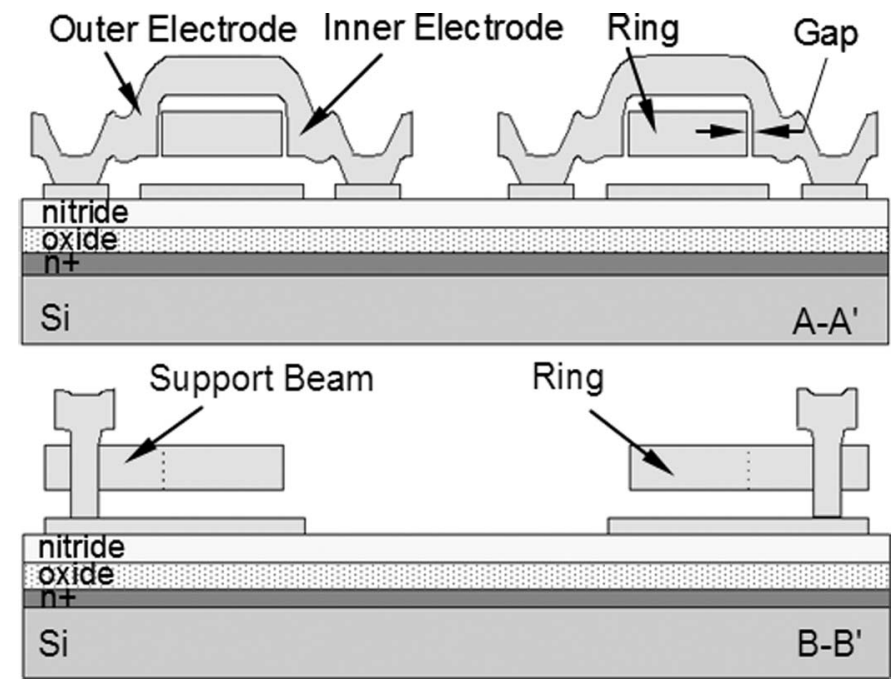

Fig. 8. Cross sections for a finished EWGR along the $A-A^{\prime}$ and $B-B^{\prime}$ planes in Fig. 1(b).

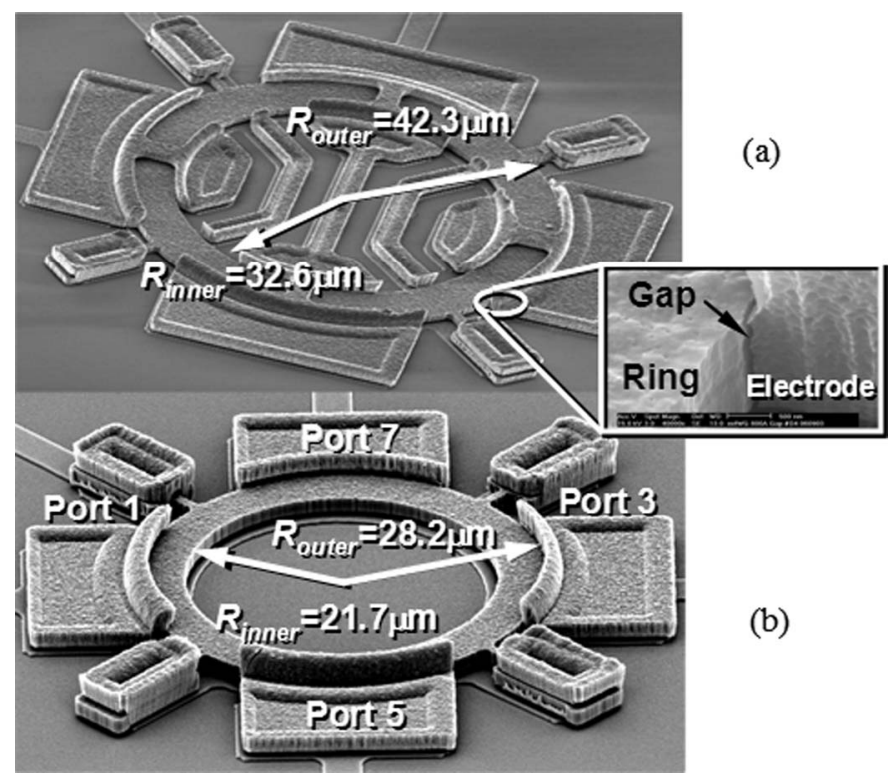

Fig. 9. Scanning electron micrographs of fabricated EWGRs: (a) 435$\mathrm{MHz}$ device; (b) 651-MHz device with only outer electrodes.

with earlier disk resonators, the yield-limiting step of this process centers around the clearing of the tiny lateral gaps during the final sacrificial hydrofluoric acid release step. The yield of operational devices was close to $85 \%$ in the University of Michigan Nanofabrication Facility.

\section{Experimental Results}

Table I summarizes the designs, models, and measurements for the EWGR devices demonstrated in this work. The devices range in frequency from $200 \mathrm{MHz}$ to more than $1.5 \mathrm{GHz}$.

A $220-\mathrm{MHz}$ device and the $435-$ and $651-\mathrm{MHz}$ devices of Fig. 9(a) and (b) were tested under controlled pressures

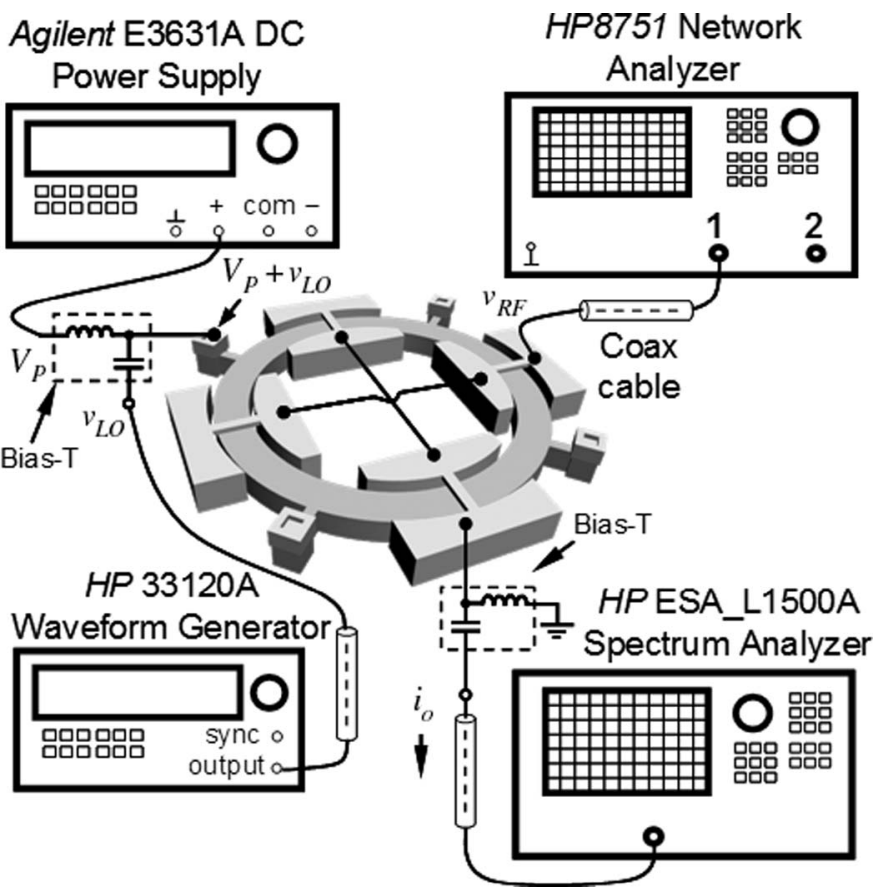

Fig. 10. Schematic illustrating the mixing measurement scheme, showing detailed connections for input excitation and output measurement instrumentation.

using a custom-built chamber with an electrical hook-up similar to Fig. 1(b), but modified for actual measurement to allow the mixing approach described in [25], [26], which greatly enhances the detectable output current relative to potentially troublesome feedthrough parasitics. In this mixing approach shown in Fig. 10, a local oscillator signal added to the dc-bias $V_{P}$ uses capacitive transducer nonlinearity to separate motional currents from feedthrough parasitics in the frequency domain. No effort was made to impedance-match devices to the measuring spectrum analyzer. This helped to preserve true mechanical $Q$ values, but at the cost of measurement mismatch loss. Of course, measurement mismatch loss should not be misinterpreted as insertion loss; indeed, if these resonators or filters constructed of them were properly terminated, it should be clear that their very high $Q$ 's will lead to very small insertion losses, as demonstrated in [8] and [27].

Fig. 11(a) and (b) present frequency characteristics for the 220-MHz device and the 435-MHz device of Fig. 9(a) measured under $200 \mu$ Torr vacuum, showing $Q$ 's of 7,700 and 6,500, respectively. Fig. 11(c) presents, on the same plot, spectra measured for the $651-\mathrm{MHz}$ resonator of Fig. 9(b) under vacuum and air using only one set of electrode quarters (i.e., drive port 1, sense from port 5); and under vacuum again, but this time using all electrodes (i.e., 1 and 3 for driving, 5 and 7 for sensing). The single electrode-quarter measurements exhibit very similar $Q$ 's of 4,650 and 4,550 in vacuum and air, respectively, thereby further demonstrating over the work of [1] that high stiffness, high frequency micromechanical resonators do not require vacuum to attain high $Q$. The measurement in vacuum using the additional electrode-quarters exhibits 
TABLE I

Extensional Wine-Glass Ring Resonator Design and Performance Summary.

\begin{tabular}{|c|c|c|c|c|c|c|c|}
\hline \multirow[b]{2}{*}{ Parameter } & \multirow[b]{2}{*}{ Source } & \multicolumn{5}{|c|}{ Designed Frequency $(3)-(5)$} & \multirow{2}{*}{$\begin{array}{l}\text { Unit } \\
\mathrm{MHz}\end{array}$} \\
\hline & & 209.96 & 425.3 & 634.6 & 1209.5 & 1519.1 & \\
\hline Ring outer radius $*, R_{\text {outer }}$ & layout & $50(50-0.47)$ & $42.3(42.3-0.12)$ & $28.2(28.2-0.08)$ & $22.2(22.2-0.01)$ & $40(40-0.01)$ & $\mu \mathrm{m}$ \\
\hline Ring inner radius ${ }^{*}, R_{\text {inner }}$ & layout & $30.5(30.5+0.47)$ & $32.6(32.6+0.12)$ & $21.7(21.7+0.08)$ & $11.8(22.2+0.01)$ & $31.8(31.8+0.01)$ & $\mu \mathrm{m}$ \\
\hline Ring thickness ${ }^{*}, t$ & layout & 2.0 & 2.0 & $2.0(2.1)$ & 2.0 & 2.0 & $\mu \mathrm{m}$ \\
\hline Electrode angle, $\theta_{2}=-\theta_{1} \quad \begin{array}{l}\text { outer } \\
\text { inner }\end{array}$ & $\begin{array}{l}\text { layout } \\
\text { layout }\end{array}$ & $\begin{array}{l}38.2 \\
32.7\end{array}$ & $\begin{array}{l}37.2 \\
27.4\end{array}$ & 35.8 & 27.6 & $\begin{array}{l}36.5 \\
34.2\end{array}$ & $\begin{array}{l}\text { deg. } \\
\text { deg. }\end{array}$ \\
\hline Support beam length, $L_{S}$ & layout & 6.6 & 4.6 & 3.8 & 2.8 & 4.4 & $\mu \mathrm{m}$ \\
\hline Support beam width, $W_{S}$ & layout & 1.6 & 1.6 & 1.6 & 1.6 & 1.6 & $\mu \mathrm{m}$ \\
\hline Electrode-to-resonator gap ${ }^{*}, d_{o}$ & measured & 63 & $85(87)$ & $85(83)$ & 85 & 63 & $\mathrm{~nm}$ \\
\hline FEM-simulated resonance freq., $f_{O}$ & FEM & 210.6 & 426.8 & 638.9 & 1220.2 & 1542.5 & $\mathrm{MHz}$ \\
\hline Measured resonance freq., $f_{O}$ & measured & 220.8 & 435.8 & 651.1 & 1212.1 & 1522.9 & $\mathrm{MHz}$ \\
\hline Quality factor, $Q$ & measured & 7,700 & 6,500 & 4,700 & 3,700 & 3,000 & - \\
\hline DC-bias, $V_{P}$ & measured & 15 & 4 & 10 & 20 & 5 & $\mathrm{~V}$ \\
\hline RF input amplitude, $v_{R F}$ & measured & 0.316 & 1 & 1 & 1.78 & 0.56 & $\mathrm{~V}$ \\
\hline LO carrier amplitude, $v_{L O}$ & measured & 10 & 4 & 10 & 10 & 5 & $\mathrm{~V}$ \\
\hline Measured output power, $P_{O}$ & measured & -54.8 & -79.1 & -77.0 & -84.0 & -85 & $\mathrm{dBm}$ \\
\hline Measured output current, $i_{O}$ & measured & 11.5 & 0.7 & 0.89 & 0.4 & 0.34 & $\mu \mathrm{A}$ \\
\hline Resonator mass, $m_{r e}$ & $(18)$ & $8.01 \times 10^{-12}$ & $3.01 \times 10^{-12}$ & $1.44 \times 10^{-12}$ & $1.786 \times 10^{-12}$ & $2.34 \times 10^{-12}$ & $\mathrm{~kg}$ \\
\hline Resonator stiffness, $k_{r e}$ & $(18)$ & $1.54 \times 10^{7}$ & $2.30 \times 10^{7}$ & $2.40 \times 10^{7}$ & $1.04 \times 10^{8}$ & $2.14 \times 10^{8}$ & $\mathrm{~N} / \mathrm{m}$ \\
\hline Damping coefficient, $c_{r e}$ & $(18)$ & $1.44 \times 10^{-6}$ & $1.84 \times 10^{-6}$ & $1.25 \times 10^{-6}$ & $3.68 \times 10^{-6}$ & $8.00 \times 10^{-6}$ & $\mathrm{~kg} / \mathrm{s}$ \\
\hline Motional resistance, $R_{x}$ & $\begin{array}{l}(22) \\
(27) \\
\text { measured }\end{array}$ & $\begin{array}{l}9.83 \\
7.84 \\
9.16\end{array}$ & $\begin{array}{l}701.0 \\
497.7 \\
712.8\end{array}$ & $\begin{array}{l}575.9 \\
347.7 \\
559.7\end{array}$ & $\begin{array}{c}1148 \\
836.8 \\
1114\end{array}$ & $\begin{array}{l}787.5 \\
508.4 \\
791.6\end{array}$ & $\mathrm{k} \Omega$ \\
\hline Inductance, $L_{x}$ & $\begin{array}{l}(22) \\
\text { measured }\end{array}$ & $\begin{array}{l}0.0546 \\
0.0508\end{array}$ & $\begin{array}{l}1.664 \\
1.692\end{array}$ & $\begin{array}{l}0.6617 \\
0.6431\end{array}$ & $\begin{array}{l}0.5577 \\
0.5412\end{array}$ & $\begin{array}{l}0.2304 \\
0.2316\end{array}$ & $\mathrm{H}$ \\
\hline Capacitance, $C_{x}$ & $\begin{array}{l}(22) \\
\text { measured }\end{array}$ & $\begin{array}{l}0.952 \times 10^{-17} \\
1.022 \times 10^{-17}\end{array}$ & $\begin{array}{l}8.016 \times 10^{-20} \\
7.883 \times 10^{-20}\end{array}$ & $\begin{array}{l}9.031 \times 10^{-20} \\
9.293 \times 10^{-20}\end{array}$ & $\begin{array}{l}3.092 \times 10^{-20} \\
3.185 \times 10^{-20}\end{array}$ & $\begin{array}{l}4.740 \times 10^{-20} \\
4.715 \times 10^{-20}\end{array}$ & $\mathrm{~F}$ \\
\hline
\end{tabular}

Numbers in () indicate adjusted values needed to match simulated quantities to measured ones (resonance frequency and motional resistance).

**For polysilicon material, $\rho=2.3 \times 10^{3} \mathrm{~kg} / \mathrm{m}^{3} ; \sigma=0.226 ; E=150 \times 10^{9} \mathrm{~Pa}$

a $10 \mathrm{~dB}$ larger transmission power than its single electrodequarter counterpart, showing the expected increase in output power with more electrodes. The motional resistance extracted from the data of Fig. 11, using the procedures in [3], is $\sim 560 \mathrm{k} \Omega$, which is substantially lower than the $4 \mathrm{M} \Omega$ previously measured for a $733-\mathrm{MHz}$ disk resonator with a radius of $10 \mu \mathrm{m}$ [3], proving the $R_{x}$-lowering ability of this design.

To verify the formulations of Section III, the predicted frequency characteristics for the EWGRs of Fig. 11 were simulated using the equivalent circuit elements summarized in Table I, which were determined using the formulations of Section III with parameter adjustments (indicated in parentheses in the table) made to match the measured curves. These predicted curves are also plotted in Fig. 11 alongside the corresponding actual data. Here, the magnitude of the parameter adjustments (e.g., inner and outer radii) needed to match theory to the actual measurements are quite small, bolstering confidence in the accuracy of Section III's formulations.

\section{A. GHz Frequencies}

Via use of higher extensional wine-glass modes, $\mathrm{GHz}$ frequencies were also achieved. Fig. 12 presents the frequency characteristic for a 1.2-GHz, 4th-mode EWGR measured in vacuum. (The 4 th mode exhibits a similar deformed ring outline as the 2nd mode of Fig. 2(a), but with more nodal circles inside the ring.) The motional resistance extracted from the data of Fig. 12 is $1.1 \mathrm{M} \Omega$, which is 2.2 times lower than the $2.4 \mathrm{M} \Omega$ previously measured for a $1.156-\mathrm{GHz}$ disk resonator with a radius of $10 \mu \mathrm{m}$ [3], again demonstrating the $R_{x}$-lowering ability of this design. And again, the mea- sured value of motional resistance matches those that were obtained via the formulation of Section III, as indicated by the closely matched theoretical curve, simulated using the circuit summarized in Table I and plotted alongside the measured data in Fig. 12. Fig. 13 presents the measured frequency characteristic for a 1.52-GHz, 4th-mode EWGR in vacuum with a $Q$ of 2,800 . This represents the highest frequency to date measured for polysilicon resonator devices. Again, Table I summarizes each of the measured designs.

\section{B. Tailoring Series Motional Resistance}

To demonstrate the degree to which the EWGR allows tailoring of the series motional resistance $R_{x}$, Fig. 14 presents measured frequency characteristics for two 510$\mathrm{MHz}$ EWGRs with different ring radius. As seen, the larger ring with an outer radius of $100 \mu \mathrm{m}$ has an $R_{x}$ of $122 \mathrm{k} \Omega$, which is 10 times smaller than the $1.18 \mathrm{M} \Omega$ measured for the smaller ring with an outer radius of $31.1 \mu \mathrm{m}$. The reduction of the $R_{x}$ matches theory and results from both a larger sensing area and a slightly higher quality factor $Q$, c.f., (27).

\section{Influence of Support Beam Design}

To verify the dependence of extensional wine-glass frequency and $Q$ on the design of its supports, a $460-\mathrm{MHz}$ EWGR was purposely designed to have much shorter support beams than those dictated by the design formulations of Section III, with a length of $2.5 \mu \mathrm{m}$ instead of the $4.46 \mu \mathrm{m}$ governed by (10) and (11). Fig. 15 presents the measured frequency characteristic for both cases plotted 

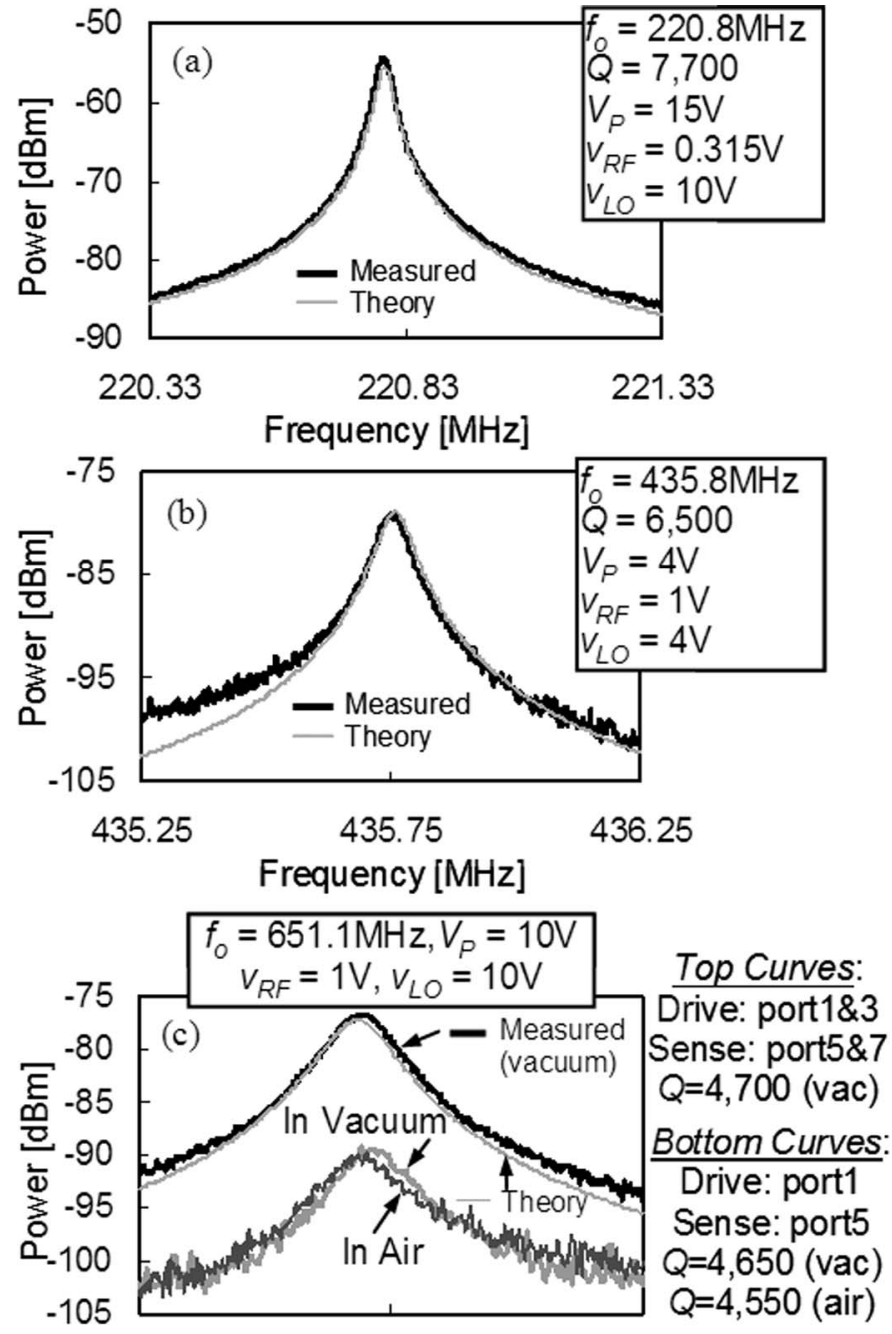

650.6

651.1

651.6

Frequency $[\mathrm{MHz}]$

Fig. 11. Frequency characteristics for EWGRs: (a) 220.8-MHz resonator; (b) 435.8- $\mathrm{MHz}$ resonator of Fig. 9(a); (c), (d), and (e) 651$\mathrm{MHz}$ resonator of Fig. 9(b). The dark curves are measured and the light curves are theoretically predicted using the corresponding equivalent circuits summarized in Table I, which were themselves generated to match measurement using the small adjustments indicated in parentheses in Table I.

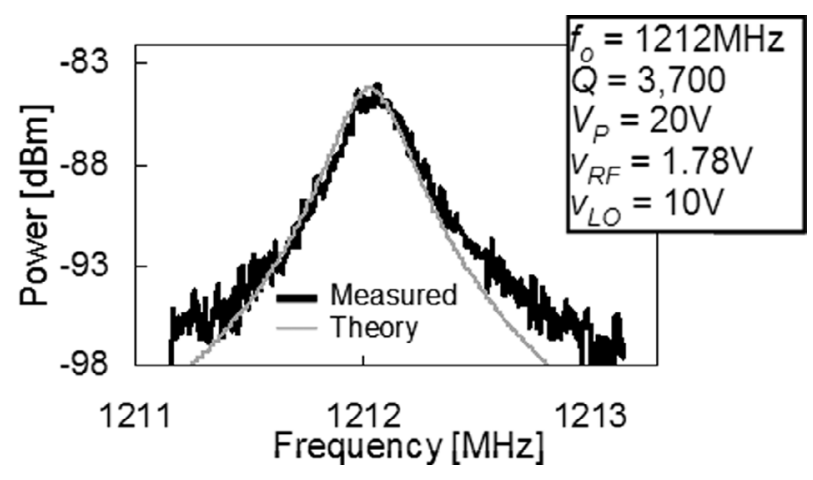

Fig. 12. Measured frequency spectrum for a $1.21-\mathrm{GHz}$, 4th-mode EWGR. The dark curve is measured and the light curve is theoretically predicted using the corresponding equivalent circuit summarized in Table I, which was itself generated to match measurement using the small adjustments indicated in parentheses in Table I.

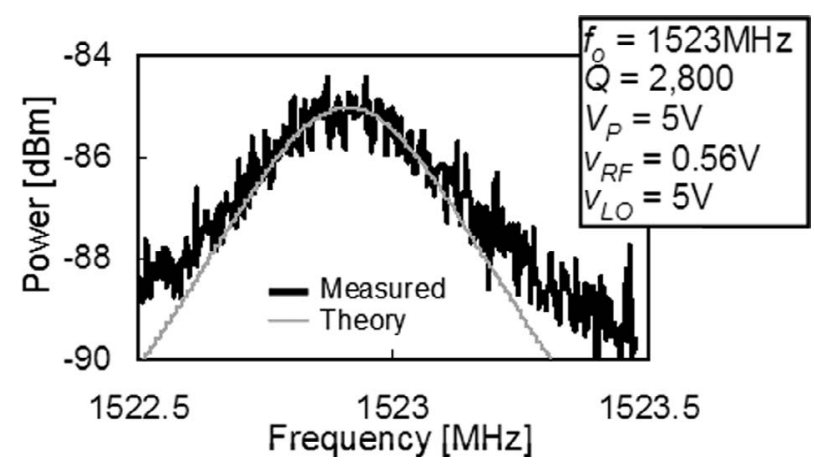

Fig. 13. Measured frequency spectrum for a $1.52-\mathrm{GHz}$, 4th-mode EWGR. The dark curve is measured and the light curve is theoretically predicted using the corresponding equivalent circuit summarized in Table I, which was itself generated to match measurement using the small adjustments indicated in parentheses in Table I.

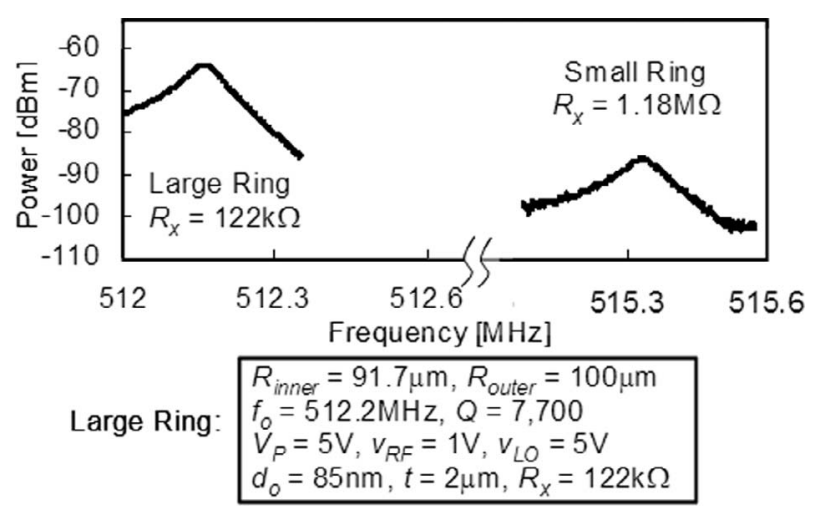

Small Ring: \begin{tabular}{l|l|}
$R_{\text {imner }}=22.9 \mu \mathrm{m}, R_{\text {outer }}=31.1 \mu \mathrm{m}$ \\
$f_{O}=515.3 \mathrm{MHz}, Q=5,350$ \\
$V_{P}=5 \mathrm{~V}, v_{R F}=1 \mathrm{~V}, v_{L O}=4 \mathrm{~V}$ \\
$d_{O}=85 \mathrm{~nm}, t=2 \mu \mathrm{m}, R_{X}=1.18 \mathrm{M} \Omega$
\end{tabular}

Fig. 14. Measured frequency characteristics for $510-\mathrm{MHz}$ EWGRs with different inner and outer radii. The left spectrum corresponds to a large ring and the right spectrum to a small ring.

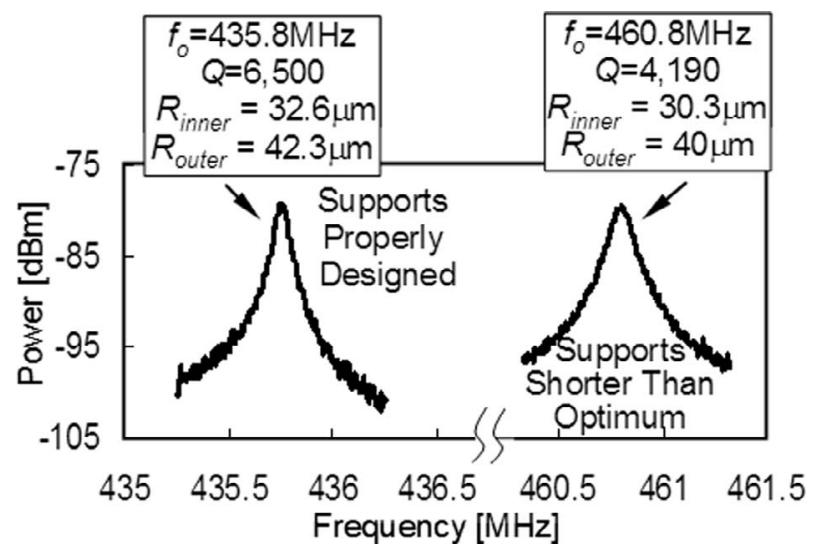

Fig. 15. Measured frequency spectra illustrating the variation of $Q$ with support beam length. 


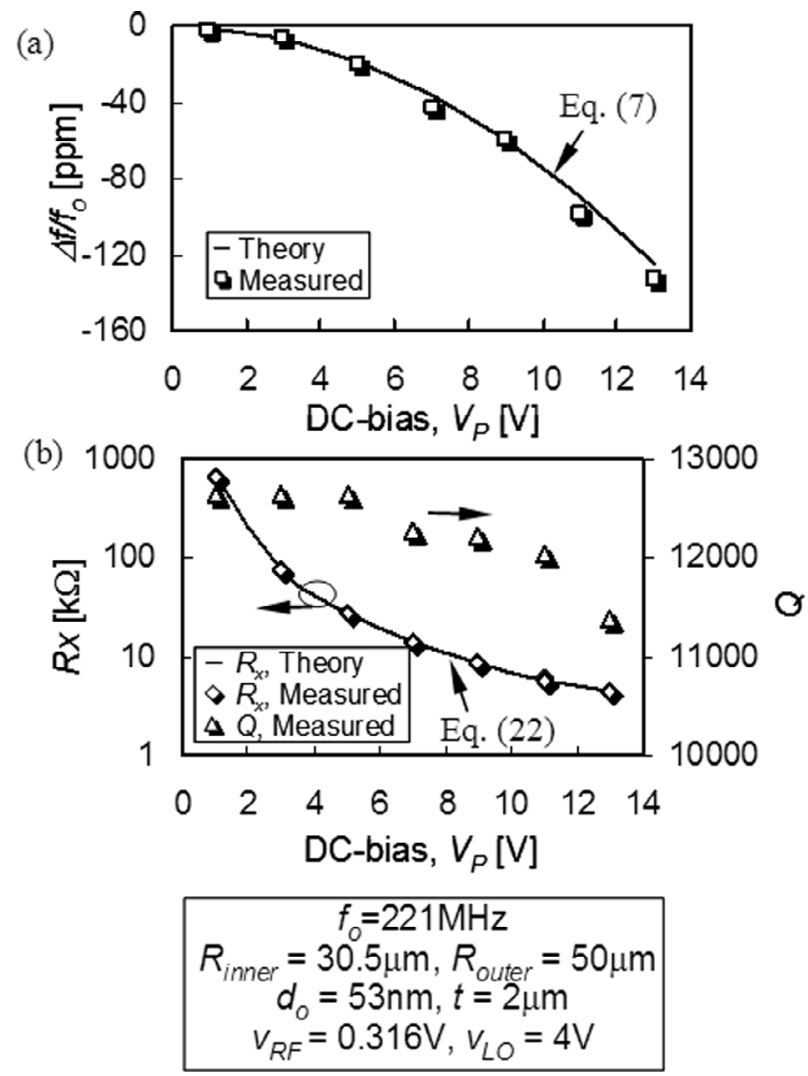

Fig. 16. Simulated and measured resonator parameters versus dcbias $V_{P}$ for a 221-MHz EWGR: (a) fractional frequency change; and (b) motional resistance $R_{x}$ and $Q$.

on the same graph. Here, the ring with properly designed supports resonates at the desired $435 \mathrm{MHz}$ frequency and exhibits a $Q$ of 6,500 ; hereas the short-support beam counterpart resonates at $460 \mathrm{MHz}$ and exhibits a $Q$ of only 4,190 , which is lower than with the properly designed support beam, but still acceptable. To bolster the confidence of these $Q$ values, two more resonators of each kind were also measured, each performing similarly to that of the Fig. 15 devices, with all three properly supported devices having an average $Q$ of 6,561 , and all three short-support devices an average $Q$ of only 4,296. This result agrees with that seen in [28], where quarter-wavelength supports for free-free beams were seen to maximize $Q$, but were not necessary to achieve high $Q$ 's $>10,000$.

\section{Frequency, $R_{x}$, and $Q$ Versus DC-Bias $V_{P}$}

To verify the influence of the electrical stiffness $k_{e}$ discussed in Section III, the shift of resonance frequency was measured at different dc-bias $V_{P}$ 's for a 221-MHz EWGR. Fig. 16(a) presents measured and theoretically predicted plots of fractional frequency change versus dc-bias $V_{P}$, showing a 130-ppm frequency shift over a $V_{P}$ range of $13 \mathrm{~V}$. Although extended frequency tunability might be achievable with an even smaller gap spacing $d_{o}$, the range of frequency tuning via $V_{P}$ is quite small, as predicted in Section III.

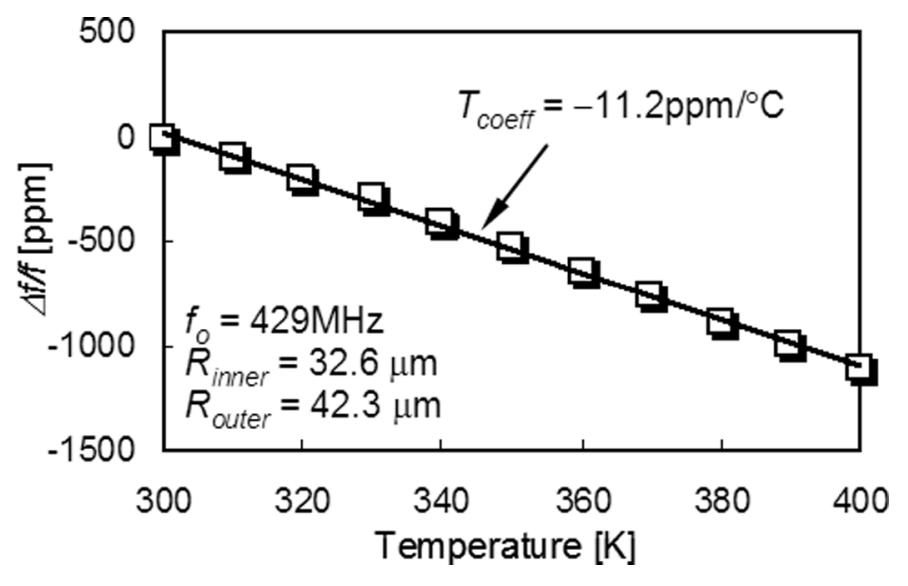

Fig. 17. Measured fractional frequency change versus temperature for a 2nd-mode, 429-MHz EWGR.

Besides the resonance frequency change, (22) and (27) predict a strong dependence of the motional resistance $R_{x}$ on the dc-bias $V_{P}$. Fig. 16(b) presents a measured curve of $R_{x}$ versus $V_{P}$ for the $221-\mathrm{MHz}$ device, showing a dramatic decrease of $R_{x}$ from hundreds of $\mathrm{k} \Omega \mathrm{s}$ to less than $5 \mathrm{k} \Omega$ as the dc-bias $V_{P}$ increases from $1 \mathrm{~V}$ to $13 \mathrm{~V}$ - a very strong dependence, indeed.

Fig. 16(b) also plots $Q$ versus $V_{P}$, showing a slight $Q$ degradation with an increase in dc-bias, in which is expected because a dc-bias shift causes a shift in total stiffness $k_{r e}$, and $Q=k_{r e} /\left(\omega_{o} c_{r e}\right)$. It should be noted that the higher-than-average $Q$ for this device (i.e., more than 10,000 here versus 7,700 for the $220-\mathrm{MHz}$ device in Table I) is a result of thinner support beams caused by undercut in the final over-etch step (polysilicon electrode definition) in the fabrication of this device.

\section{E. Temperature Dependence}

Fig. 17 presents a measured plot of fractional frequency change versus temperature for a $429-\mathrm{MHz}$ EWGR operated in its 2nd mode. The uncompensated temperature coefficient of $-11.2 \mathrm{ppm} /{ }^{\circ} \mathrm{C}$ is somewhat better than the temperature coefficient exhibited by previous polysilicon resonator designs [1], [16]. In addition, the curve is linear and monotonically decreasing with temperature, making it more amenable to circuit-based compensation than the quadratic dependency often exhibited by quartz crystal counterparts.

\section{Spurious Modes And Their Suppression}

Unfortunately, although capable of achieving lower impedance than solid disk resonators [1], the EWGR and a later radial-mode "hollow-disk" ring introduced in [2] both suffer from the presence of other bulk resonance modes close to their own intended resonances. The close proximity of these "spurious" modes is no surprise because they derive from the same set of equations that govern the "desired" extensional wine-glass (EWG) mode. Eqs. (3)-(5) 

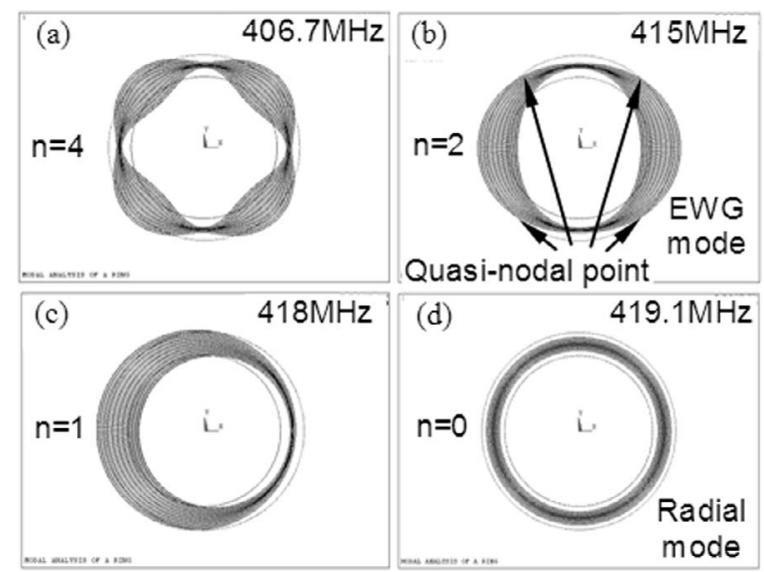

Fig. 18. ANSYS simulated mode shapes of a ring resonator with an inner radius of $32.4 \mu \mathrm{m}$ and an outer radius of $42.3 \mu \mathrm{m}$. Here, (b) is the desired mode, while $(\mathrm{a})(\mathrm{c})(\mathrm{d})$ are spurious.

are in fact a universal set of equations for the resonance frequency of in-plane vibrations of ring shaped resonators. In particular, each possible ring resonance mode is represented by the circumferential number $n$ in (5), which when equal to 2, defines the extensional wine-glass mode of this work; but when equal to 0 , defines the hollow-disk mode of [2]. In general, the higher $n$ becomes, i.e., the farther away from 0 or 2 , the farther away the resonance frequency of its corresponding mode from the $n=0$ and $n=2$ modes. To limit the present discussion to local modes, only the $n=1$ and $n=4$ modes are examined in depth here. Setting the circumferential number $n$ in (5) to 0,1 , and 4, respectively; plugging in the values of $R_{\text {inner }}$ and $R_{\text {outer }}$ for $n=2$ (i.e., for the compound $(2,2)$ or "extensional wineglass" mode); then solving (3) and (4), yields the resonance frequency $f_{o}$ corresponding to $n=0$ (i.e., the compound $(0,2)$ or "hollow-disk" mode), $n=1$ (i.e., the compound $(1,2)$ or "eccentric ring" mode), and $n=4$ (i.e., the compound (4,2) or "square" mode). Fig. 18(a), (c), and (d) depict each of these troublesome lateral spurious modes, with theoretically predicted frequencies as close as $3 \mathrm{MHz}$ to the intended $415 \mathrm{MHz}$ extensional wine-glass frequency for a ring with an inner radius $R_{\text {inner }}$ of $32.4 \mu \mathrm{m}$ and an outer radius $R_{\text {outer }}$ of $42.3 \mu \mathrm{m}$.

To make matters worse, the separation between intended and spurious modes becomes even smaller as the ring radius increases - a condition that seems to undermine the radius-dependent impedance advantage of a ring structure. For example, (3)-(5) predict that for an even larger ring with an outer radius of $100 \mu \mathrm{m}$, the extensional wine-glass and radial modes are only $0.7 \mathrm{MHz}$ apartquite problematic if large ring radius is to be used to reduce device impedance. Fig. 19 presents a simulated plot identifying the different modes predicted by (3)-(5) at frequency locations in close proximity to the desired extensional wine-glass mode of this work.

Perhaps the best defense against the above problem is to eliminate the undesired modes from the outset. This work attempts to do so by: 1) designing resonator sup-

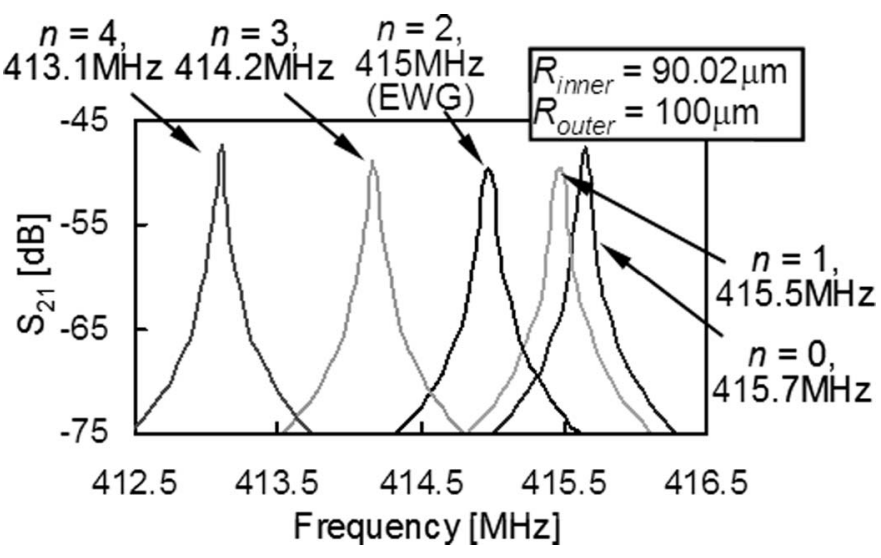

Fig. 19. Simulated plot showing the frequencies of different modes close to the extensional wine-glass mode $(n=2)$.

ports to suppress spurious modes; 2) applying forces that accentuate the desired mode shape (in this case, the extensional wine-glass mode shape) while opposing the shapes of other modes; and 3) using a detection scheme that cancels the motional currents of undesired modes. Each of these strategies is now described.

\section{A. Mechanical Mode Suppression (Mode Damping)}

As has been shown numerous times, the $Q$ of any high-stiffness, high-frequency resonator is a strong function of the energy per cycle lost through its supports and anchors. Indeed, the micromechanical resonators that presently hold frequency- $Q$ product records at VHF and UHF all do so via isolating support designs that suppress anchor losses [1], [2], [16], [23]. Here, quarter-wavelength support design is often used to reflect energy attempting to escape the resonator through its anchors back into the resonator, allowing the device to operate with its maximum energy per cycle, hence, maximum $Q$. The opposite approach is also possible, where supports are designed to realize low impedance conduits through which energy from unwanted mode shapes can escape through anchors, lowering the $Q$ 's of these modes, and thereby suppressing them. Clearly, support design to minimize anchor losses for the desired mode, while maximizing such losses for other mode shapes, constitutes one of the more effective approaches to selecting a desired mode while suppressing others.

The design of Fig. 1(b) does just this by attaching its support tethers to the quasi-nodal points of the EWGR and suppressing unwanted modes by physically attenuating their motions. The degree to which other modes can be attenuated depends on how rigid the tethers appear to their specific motions at their specific frequencies. For example, mode (a) is attenuated the most by the support design of Fig. 1(b) because the tether attachment locations actually correspond to its anti-nodes (i.e., its points of maximum displacement). This mode, in fact, is not even measurable in any of the plots to be presented in Fig. 23. From Fig. 18, modes (c) and (d) have mode shapes that, again, oppose the support structure, but to a lesser degree than mode (a). 
Mode (d), the radial mode, will be most strongly suppressed if the support beams have very small lengths (e.g., less than $1 / 8$-wavelength) or lengths corresponding to an extensional half-wavelength at the radial-mode resonance frequency. In the present design, the support beams are sized to accentuate the extensional wine-glass mode (as opposed to attenuate the radial mode). In particular, as mentioned in Section III, although the support attachment points correspond to extensional wine-glass nodes in the radial direction, they are actually not rotational nodes, so rotations still occur at these points. To isolate this rotational motion from the anchors, the support beams are designed to vibrate in a simple-fixed flexural mode at the extensional wine-glass resonance frequency. At $415 \mathrm{MHz}$, this entails a support beam length and width of $4.2 \mu \mathrm{m}$ and $1.3 \mu \mathrm{m}$, respectively. At the $419.1-\mathrm{MHz}$ radial mode resonance frequency, these dimensions correspond to an extensional-mode $\lambda / 5$ - not the half-wavelength required to suppress the radial mode completely, but still enough to provide some attenuation.

In the meantime, the support configuration of Fig. 1(b) attenuates mode (c) even less than it does (d). In particular, although mode (c)'s radial displacements are opposed somewhat by the support tethers, its rotational displacements are not opposed as strongly because these rotations are not dissimilar to those of the extensional wine-glass mode. Thus, mode (c) must be attenuated by some other means.

\section{B. Electrical Mode Suppression}

In addition to mechanical damping, electrical means for mode selection are also available. In particular, the phasings between drive electrodes and positioning/biasing of sense electrodes can accentuate one mode while suppressing others. To illustrate, Fig. 20 contrasts three different excitation/detection schemes for the extensional wineglass mode in order of increasing ability to suppress spurious modes.

The configuration of Fig. 20(a) drives via the $y$-axis electrodes and senses along the $x$-axis electrodes. This results in drive forces phase-consistent with all modes of Fig. 18 so this electrode configuration makes no attempt to suppress undesired modes by force tailoring. It, however, does suppress modes (a) and (c) via a sense electrode configuration that cancels their motional currents. In particular, the symmetry of mode (a)'s shape leads to a total capacitance along each sense electrode that remains approximately constant as the ring vibrates, making $d C / d t \sim 0$, for which the output current is zero according to (2). Furthermore, the mode shape of (c) is such that when a positive $d C / d t$ is generated at the left electrode, an approximately equal but negative $d C / d t$ ensues at the right side, creating equal and opposite motional currents given by (2) that cancel when combined at the sense terminal. The only modes that remain unscathed under this electrode-sensing configuration are those for which the $d C / d t$ 's over the left and right sense electrodes remain finite and identical at
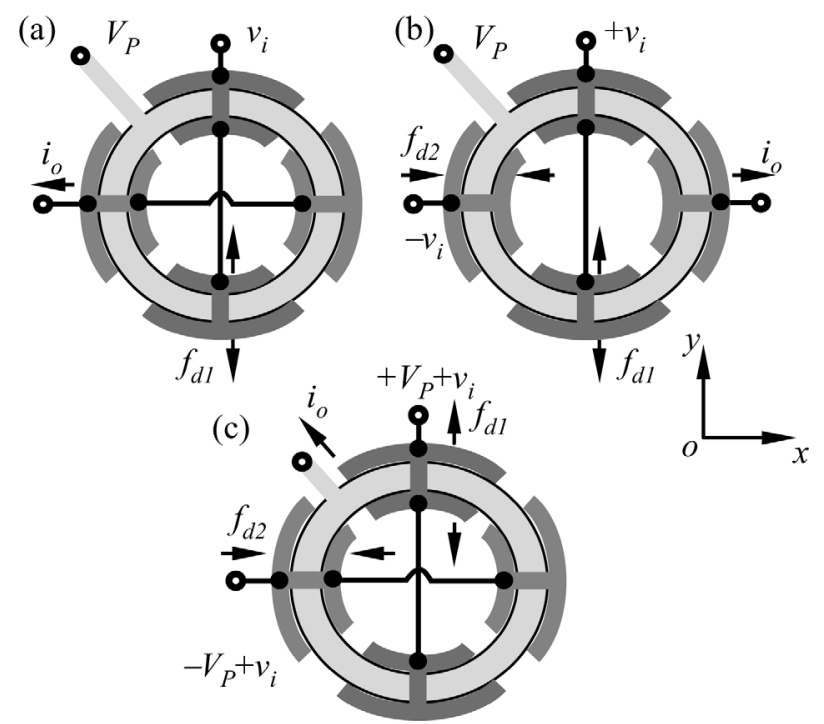

Fig. 20. Excitation/detection scheme: (a) traditional two-port; (b) orthogonal asymmetric differential drive; (c) orthogonal differential drive, common-mode one-port sense, where the output is taken from the structure itself.

all times, creating a condition where currents from each electrode add at the sense terminal. Fortunately, this includes the desired extensional wine-glass mode shape of Fig. 18(b). Unfortunately, it also includes the unwanted radial mode $(\mathrm{d})$.

To suppress mode (d), the configuration of Fig. 20(b) uses an orthogonal differential input, with $+v_{i}$ applied to the $y$-axis electrodes, and $-v_{i}$ to the left side $x$-axis electrode, to tailor its force phasings so that they oppose some or all of the displacement directions of modes (a) and (d), thereby suppressing them. Unfortunately, with the right side $x$-axis electrode used for sensing, the $x$-axis $-v_{i}$ drive component is not fully symmetric, so it does little to suppress mode (c). Furthermore, the right side $x$-axis sense electrode in Fig. 20(b) does nothing to cancel mode (c)'s motional current.

To remedy this problem, the (one-port) scheme of Fig. 20(c) achieves an orthogonal fully differential forcing and sensing configuration by applying $+V_{P}+v_{i}$ to the $y$-axis electrodes, $-V_{P}+v_{i}$ to the $x$-axis electrodes, and using the ring itself as the current output port (which normally makes it an effective ac ground). With this $V_{P}$ configuration, according to the excitation force expression (1), $y$-axis electrodes generate forces that have the same amplitude but opposite directions (inward and outward, respectively) as those generated by the (orthogonal) $x$-axis electrodes, effectively imparting an alternative pulling or compression on the ring. In effect, a differential force acting along orthogonal axes is attained using a single phase $v_{i}$ and $\mathrm{a} \pm V_{P}$. This orthogonal, fully differential-forcing configuration now suppresses all spurious modes (a)(c)(d), while accentuating the extensional wine-glass mode. The scheme of Fig. 20(c) not only attenuates via drive force tailoring, but also further suppresses spurious modes via its one-port common-mode sensing configuration. Here, the 


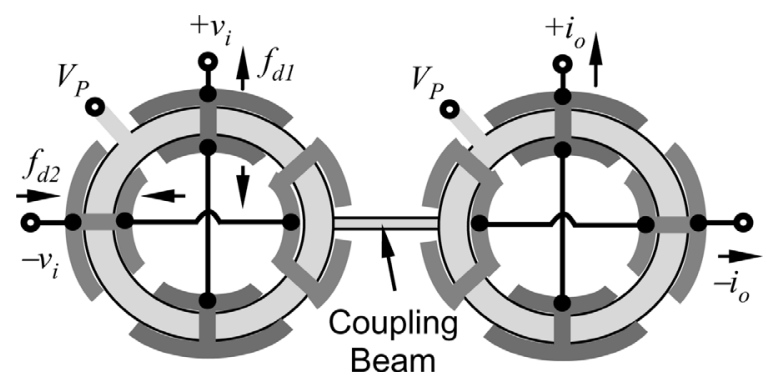

Fig. 21. Schematic of a coupled two-resonator EWGR filter, illustrating differential input/output and bias electrode configurations.

output current is sensed directly off the ring, and $\pm V_{P}$ 's are used to cancel the output currents of any $y$-axis asymmetric mode shape, such as mode (c), while preserving those of the extensional wine-glass mode.

It should be noted that when used in an actual, fully differential application, such as the filter in [27], a commonmode sense output is generally not needed. Rather, as shown in the example ring filter circuit of Fig. 21, an orthogonal differential drive and sense can be used, where $+v_{i}$ and $-v_{i}$ are applied along orthogonal axes at the input port, $V_{P}$ is directly applied to the resonator, and the output is taken differentially using the orthogonal electrodes of the output resonator.

\section{Measured Verification of Mode Suppression}

To evaluate the efficacy of the described spurious modesuppression techniques, each technique was applied to a fabricated 415-MHz EWGR, and measurements were made under vacuum using the previously described custom-built vacuum chamber. To facilitate output current detection, the configurations of Fig. 20(a)-(c) were modified for actual measurement to allow the mixing measurement approach described in [25], [26], which greatly enhances the detectable output current relative to potentially troublesome feedthrough parasitics. In this mixing approach, a local oscillator signal $v_{L O}$ added to the dc-bias $V_{P}$ uses capacitive transducer nonlinearity to allow excitation of the resonator via an out-of-band input signal $v_{R F}$. In particular, when the electrical signal $\left(V_{P}+v_{R F}+v_{L O}\right)$ is applied across an electrode-to-resonator gap, nonlinear mixing of the two electrical signals $v_{R F}$ and $v_{L O}$ gives rise to a force component

$$
F_{\text {mix }}=-\frac{1}{2} V_{R F} V_{L O} \cdot \frac{\partial C_{j}}{\partial r} \cos \left[\left(\omega_{R F} \pm \omega_{L O}\right) t\right],
$$

where $\omega_{R F}$ and $\omega_{L O}$ are the radian frequencies, and $V_{R F}$ and $V_{L O}$ are the amplitudes, of $v_{R F}$ and $v_{L O}$, respectively; and where $F_{\text {mix }}$ becomes an in-band component capable of driving the resonator into resonance when $\left(\omega_{R F}-\omega_{L O}\right)$ or $\left(\omega_{R F}+\omega_{L O}\right)$ is equal to the resonance frequency $\omega_{o}$. By exciting the device with inputs that are all out-of-band, this mixing measurement method effectively separates the input excitation signal (and its associated feedthrough parasitics) from in-band output motional currents in the



Fig. 22. Mixing measurement setups for each setup in Fig. 20.

frequency domain, allowing interference-free detection. It should be noted that although the dc-bias $V_{P}$ does not appear in the above force (28), it is still needed if the output current given by (2) is to be sensed at the device resonance frequency.

Fig. 22 presents the mixing-based configurations corresponding to each of the configurations in Fig. 20(a)-(c). In each of these, the $V_{P}$ in the Fig. 20 version can merely be replaced with $v_{L O}+V_{P}$, and the $v_{i}$ by $v_{R F}$, while retaining all signs, i.e., $-V_{P}$ is replaced by $-\left(v_{L O}+V_{P}\right)$. But note that in Fig. 22(c), although $v_{R F}-\left(v_{L O}+V_{P}\right)$ would have worked just fine for the left-hand side electrode, the minus sign has been switched from $v_{L O}$ to $v_{R F}$ (which retains the same phasings) for ease of actual implementation.

Fig. 23(a)-(c) present frequency spectra measured using the corresponding lettered configuration in Fig. 22. As predicted, the traditional two-port configuration of Fig. 22(a) excites and senses the extensional wine-glass mode of Fig. 18(b), while suppressing completely all other modes, except for the radial mode of Fig. 18(d), which is still seen but suppressed somewhat by the support structure. The frequency spectrum of Fig. 23(b) further verifies the previous prediction that the orthogonal asymmetric differential excitation scheme with single-ended output of Fig. 22(b) will suppress mode (a) and (d), but not mode (c). Finally, the absence of any spurious modes in Fig. 23(c) verifies that the orthogonal, fully differentialdrive configuration of Fig. 22(c) works as advertised to eliminate all close-in spurious modes, while accentuating the desired extensional wine-glass mode. Fig. 24 presents a measurement over a much wider frequency span from $300 \mathrm{MHz}$ to $500 \mathrm{MHz}$ (a $50 \%$ bandwidth region) using Fig. 22(c), over which no other modes are observed - an impressive demonstration of mode suppression.

\section{CONCLusions}

The demonstration by this work of extensional wineglass-mode ring resonators with frequencies as high as 


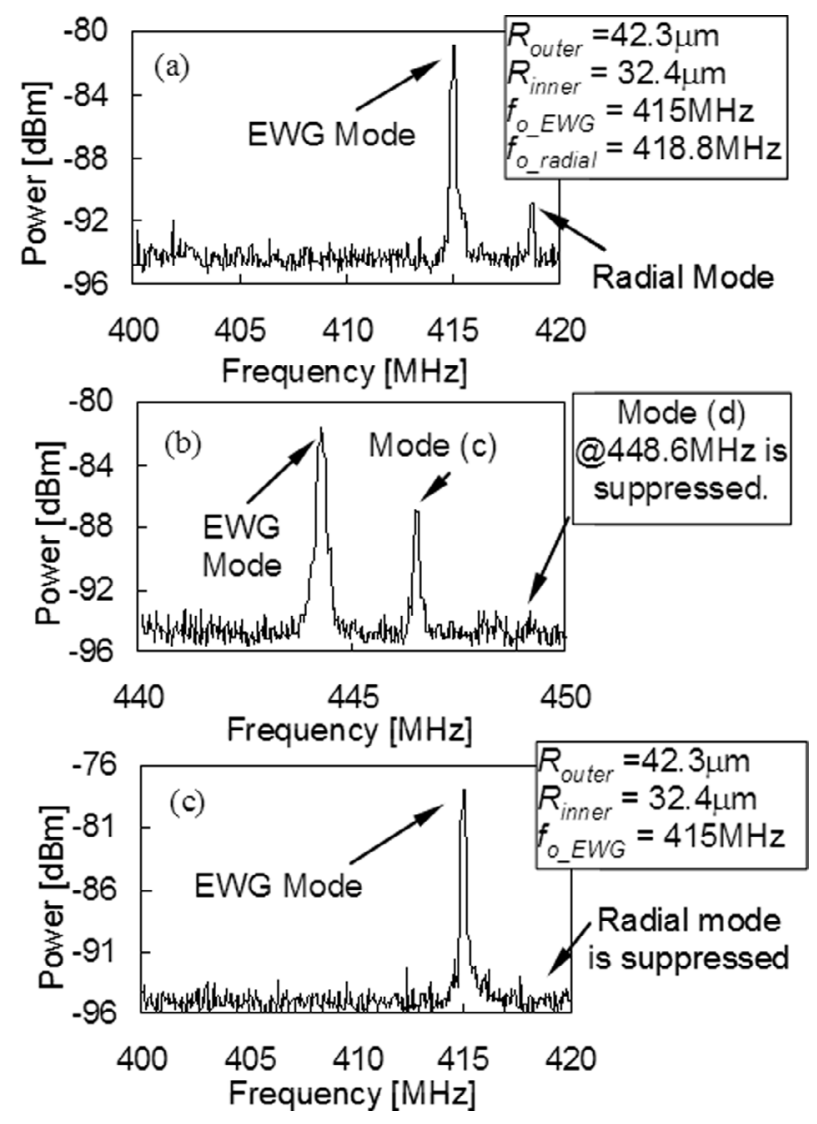

Fig. 23. Frequency spectra measured using corresponding configurations in Fig. 22. The measured resonator in (b) with five ports available is not the same design as in the other two plots, because that device did not have sufficient electrode flexibility to allow the hookup of Fig. 22(b).

1.52-GHz, $Q$ 's $>2,800$, and impedances potentially amenable to image-reject filtering and oscillator applications, constitutes a substantial step toward a capacitively transduced micromechanical resonator technology that can satisfy the RF front-end requirements of today's wireless communications. Although impedances low enough for direct connection with antennas were not demonstrated in this work, design paths based on the extensional wineglass ring structure have been identified that should enable antenna-amenable impedances in devices that retain capacitive transducers, and hence retain all their design, $Q$, and reconfigurability advantages [14]. In particular, substantially lower impedance is expected for designs that reduce electrode-to-resonator gap spacing, increase the structural film thickness, and increase the average ring radius, relative to those of the resonators demonstrated here, provided the troublesome spurious modes can be properly suppressed using the techniques described in this work. Resonator-arraying approaches [15] using the device of this work should also enable even lower motional impedances, as well as higher power handling, perhaps sufficient for transmit applications. Research to further reduce impedance in this manner is underway.



Fig. 24. Frequency spectrum measured using the setup of Fig. 22(c) over a wide frequency span, showing only the desired mode and no spurious modes.

\section{REFERENCES}

[1] J. Wang, Z. Ren, and C. T.-C. Nguyen, "1.51-GHz nanocrystalline diamond micromechanical disk resonator with materialmismatched isolating support," in Proc. 17th Int. IEEE Micro Electro Mech. Syst. Conf. (MEMS '04), Maastricht, The Netherlands, Jan. 25-29, 2004, pp. 641-644.

[2] S.-S. Li, Y.-W. Lin, Y. Xie, Z. Ren, and C. T.-C. Nguyen, "Micromechanical 'hollow-disk' ring resonators," in Proc. 17th Int. IEEE Micro Electro Mech. Syst. Conf. (MEMS '04), Maastricht, The Netherlands, Jan. 25-29, 2004, pp. 821-824.

[3] J. Wang, Z. Ren, and C. T.-C. Nguyen, "1.156-GHz self-aligned vibrating micromechanical disk resonator," IEEE Trans. Ultrason., Ferroelect., Freq. Contr., vol. 51, no. 12, pp. 1606-1628, Dec. 2004.

[4] G. Piazza, P. J. Stephanou, J. M. Porter, M. B. J. Wijesundara, and A. P. Pisano, "Low motional resistance ring-shaped contour-mode aluminum nitride piezoelectric micromechanical resonators for UHF applications," in Proc. 18th Int. IEEE Micro Electro Mech. Syst. Conf. (MEMS '05), Miami, FL, Jan. 30-Feb. 3, 2005, pp. 20-23.

[5] L. Yan, J. Wu, and W. C. Tang, "A $1.14 \mathrm{GHz}$ piezoelectrically transduced disk resonator," in Proc. 18th IEEE Int. Micro Electro Mech. Syst. Conf., Miami, FL, Jan. 30-Feb. 3, 2005, pp. 203-206.

[6] S. Humad, R. Abdolvand, G. K. Ho, G. Piazza, and F. Ayazi, "High frequency micromechanical piezo-on-silicon block resonators," in Dig. Tech. Papers, 2003 IEEE Int. Electron Devices Meeting, Washington, DC, Dec. 8-10, 2003, pp. 957-960.

[7] R. A. Johnson, Mechanical Filters in Electronics. New York: Wiley, 1983.

[8] F. D. Bannon, III, J. R. Clark, and C. T.-C. Nguyen, "High$Q$ HF microelectromechanical filters," IEEE J. Solid-State Circuits, vol. 35, no. 4, pp. 512-526, Apr. 2000.

[9] M. U. Demirci and C. T.-C. Nguyen, "A low impedance VHF micromechanical filter using coupled-array composite resonators," in Dig. Tech. Papers, 13th Int. Conf. on Solid-State Sensors 85 Actuators (Transducers'05), Seoul, Korea, June 59, 2005, pp. 2131-2134.

[10] S.-S. Li, Y.-W. Lin, Z. Ren, and C. T.-C. Nguyen, "An MSI micromechanical differential disk-array filter," in Dig. Tech. Papers, 14th Int. Conf. on Solid-State Sensors 85 Actuators (Transducers'07), Lyon, France, June 11-14, 2007, pp. 307-311.

[11] D. Weinstein, H. Chandrahalim, L. F. Cheow, and S. A. Bhave, "Dielectrically transduced single-ended to differential MEMS filter," in Proc. IEEE Int. Solid-State Circuits Conf. (ISSCC), San Francisco, CA, Feb. 5-9, 2006, pp. 318-319.

[12] P. J. Stephanou, G. Piazza, C. D. White, M. B. J. Wijesundara, and A. P. Pisano, "Design of novel mechanical coupling for contour mode piezoelectric RF MEMS filters," in Dig. Tech. Papers, Int. Conf. MEMS (iMEMS), Singapore, May 9-12, 2006, pp. 342-349.

[13] S.-S. Li, Y.-W. Lin, Y. Xie, Z. Ren, and C. T.-C. Nguyen, "Small percent bandwidth design of a 431-MHz notch-coupled micromechanical hollow-disk ring mixer-filter," in Proc. IEEE Ultrason. Symp., Rotterdam, The Netherlands, Sep. 18-21, 2005, pp. 1295-1298. 
[14] C. T.-C. Nguyen, "MEMS technology for timing and frequency control (invited)," IEEE Trans. Ultrason., Ferroelect., Freq. Contr., vol. 54, no. 2, pp. 251-270, Feb. 2007.

[15] M. U. Demirci, M. A. Abdelmoneum, and C. T.-C. Nguyen, "Mechanically corner-coupled square microresonator array for reduced series motional resistance," in Dig. Tech. Papers, 12th Int. Conf. on Solid-State Sensors \& Actuators (Transducers 'O3), Boston, MA, June 8-12, 2003, pp. 955-958.

[16] M. A. Abdelmoneum, M. U. Demirci, and C. T.-C. Nguyen, "Stemless wine-glass-mode disk $\mu$ mechanical resonators," in Proc. 16th Int. IEEE Micro Electro Mechanical Systems Conf. (MEMS '03), Kyoto, Japan, Jan. 19-23, 2003, pp. 698-701.

[17] Z. Hao, S. Pourkamali, and F. Ayazi, "VHF single-crystal silicon elliptic bulk-mode capacitive disk resonators-Part I: Design and modeling," IEEE/ASME, J. Microelectromech. Syst., vol. 13, no. 6, pp. 1043-1053, Dec. 2004.

[18] B. Bircumshaw, G. Liu, H. Takeuchi, T.-J. King, R. Howe, O. O'Reilly, and A. Pisano, "The radial bulk annular resonator: Towards a $50 \Omega$ RF MEMS filter," in Dig. Tech. Papers, 12th Int. Conf. on Solid-State Sensors \&5 Actuators (Transducers '03), Boston, MA, June 8-12, 2003, pp. 875-879.

[19] Y. Xie, S.-S. Li, Y.-W. Lin, Z. Ren, and C. T.-C. Nguyen, "UHF micromechanical extensional wine-glass mode ring resonators," in Dig. Tech. Papers, 2003 IEEE Int. Electron Devices Meeting, Washington, DC, Dec. 8-10, 2003, pp. 953-956.

[20] Y.-W. Lin, S.-L. Lee, S.-S. Li, Y. Xie, Z. Ren, and C. T.-C. Nguyen, "Series-resonant VHF micromechanical resonator reference oscillators," IEEE J. Solid-State Circuits, vol. 39, no. 12, pp. 2477-2491, Dec. 2004.

[21] T. Takano, H. Hirata, and Y. Tomikawa, "Analysis of nonaxisymmetric vibration mode piezoelectric annular plate and its application to an ultrasonic motor," IEEE Trans. Ultrason., Ferroelect., Freq. Contr., vol. 37, no. 6, pp. 558-565, Nov. 1990.

[22] H. Nathanson, W. E. Newell, R. A. Wickstrom, and J. R. Davis, Jr., "The resonant gate transistor," IEEE Trans. Electron. Dev., vol. ED-14, pp. 117-133, Mar. 1967.

[23] K. Wang, A.-C. Wong, and C. T.-C. Nguyen, "VHF free-free beam high- $Q$ micromechanical resonators," IEEE/ASME, J. Microelectromech. Syst., vol. 9, no. 3, pp. 347-360, Sep. 2000.

[24] R. D. Blevins, Formulas for Natural Frequency and Mode Shape. Malabar, FL: R.E. Krieger, 1984.

[25] A.-C. Wong and C. T.-C. Nguyen, "Micromechanical mixerfilters ('mixlers')," IEEE/ASME, J. Microelectromech. Syst., vol. 13, no. 1, pp. 100-112, Feb. 2004.

[26] J. R. Clark, W.-T. Hsu, M. A. Abdelmoneum, and C. T.-C. Nguyen, "High- $Q$ UHF micromechanical radial contour-mode disk resonators," IEEE/ASME, J. Microelectromech. Syst., vol. 14, no. 6, pp. 1298-1310, Dec. 2005.

[27] K. Wang and C. T.-C. Nguyen, "High-order medium freq. micromechanical electronic filters," IEEE/ASME, J. Microelectromech. Syst., vol. 8, no. 4, pp. 534-557, Dec. 1999.

[28] M. U. Demirci and C. T.-C. Nguyen, "Higher-mode free-free beam micromechanical resonators," in Proc. IEEE Int. Freq. Contr. Symp., Tampa, FL, May 5-8, 2003, pp. 810-818.

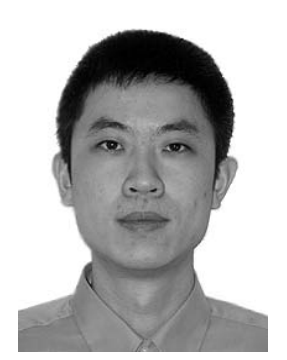

Yuan Xie (S'03-M'08) received the B.S. degree in mechanical engineering from Tsinghua University, Beijing, China, in 1998. He received the M.S. and Ph.D. degrees from the University of Michigan, Ann Arbor, in 2000 and 2006, respectively, both in electrical engineering and computer science. His $\mathrm{Ph}$.D. research focused on the development of UHF micromechanical resonators and filters for wireless communication applications. In 2006, he joined Avago Technologies, San Jose, CA, where he is currently a design engineer for the research and development of RF Film Bulk Acoustic Resonator (FBAR) duplexers and multiplexers for third-generation (3G) mobile communication applications.

Dr. Xie received the Roger A. Haken Best Student Paper Award at the 2003 IEEE International Electron Devices Meeting.



Sheng-Shian Li (S'04-M'07) received the B.S. and M.S. degrees in mechanical engineering from the National Taiwan University in 1996 and 1998, respectively. He received the M.S. and Ph.D. degrees from the University of Michigan, Ann Arbor, in 2004 and 2007 , respectively, both in electrical engineering and computer science. His doctoral work focused on micromechanical resonators and filters targeted for wireless communication applications.

From 1998 to 2000, he served in the ROC Army as a lieutenant of brigade logistics. From 2000 to 2001, he was a teaching assistant at National Taiwan University. In 2007, he joined RF Micro Devices, Greensboro, NC, where he is currently a senior design engineer for the research and development of MEMS resonators and filters.

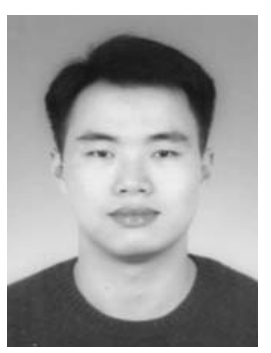

Yu-Wei Lin (S'03) was born in Taipei, Taiwan. He received the B.S. and M.S. degrees in electrical engineering from the National Taiwan University, Taipei, Taiwan, in 1997 and 1999, respectively, and the Ph.D. degree in electrical engineering from the University of Michigan, Ann Arbor, in 2007.

His master's research involved the design and testing of memory- and analog-integrated circuits. From 1999 to 2001, he served in the Army of the Republic of China as a second lieutenant to maintain wireless communications for the military. His $\mathrm{Ph}$.D. research was on developing low-phase noise micromechanical reference oscillators, which involves mixedsignal integrated circuit design and MEMS device design and fabrication for wireless communication applications. In 2007, he joined Broadcom Corporation as a staff scientist focusing on IC design for wireless connectivity.



Zeying Ren (M'04) received the B.S. and M.S. degrees in electrical engineering from Tianjin University, P.R., China, in 1987 and 1990, respectively.

From 1990 to 1998, she was a process engineer in the National Research Center for Optoelectronics (NCOT) in the Institute of Semiconductor at the Chinese Academy of Science. She was employed as a research scholar in the Department of Electrical and Computer Engineering at Northwestern University from 1998 through 2000.

From 2001 to 2002, she was employed at Nanovation Technologies as process engineer. In 2002, she joined the Solid State Electronics Laboratory as an engineer in research in the Department of Electrical Engineering and Computer Science at the University of Michigan, Ann Arbor, where she presently conducts MEMS fabrication.

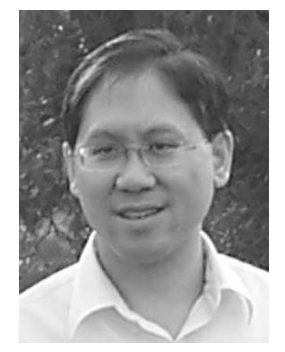

Clark T.-C. Nguyen (S'90-M'95-SM'01F'07) received the B.S., M.S., and Ph.D. degrees from the University of California at Berkeley in 1989, 1991, and 1994, respectively, all in electrical engineering and computer sciences.

In 1995, he joined the faculty of the University of Michigan, Ann Arbor, where he was a professor in the Department of Electrical Engineering and Computer Science up until mid-2006. In 2006, he joined the Department of Electrical Engineering and Computer Sciences at the University of California at Berkeley, where he is presently a professor and a co-director of the Berkeley Sensor \& Actuator Center. His research interests focus on micro electromechanical systems 
(MEMS) and include integrated micromechanical signal processors and sensors, merged circuit/micromechanical technologies, RF communication architectures, and integrated circuit design and technology. From 1995 to 1997, he was a member of the National Aeronautics and Space Administration (NASA) New Millennium Integrated Product Development Team on Communications, which roadmapped future communications technologies for NASA use into the turn of the century. In 2001, Prof. Nguyen founded Discera, Inc., a company aimed at commercializing communication products based on MEMS technology, with an initial focus on the vibrating micromechanical resonators pioneered by his research in past years. He served as vice president and chief technology officer (CTO) of Discera until mid-2002, at which point he joined the Defense Advanced Research Projects Agency (DARPA) on an IPA, where he served for 3.5 years as the program manager of the MEMS, Micro Power Generation (MPG), Chip-Scale Atomic Clock (CSAC), MEMS Exchange (MX), Harsh Environment Robust Micromechanical Technology (HERMIT), Micro Gas Analyzers (MGA), Radio Isotope Micropower Sources (RIMS), RF MEMS Improvement (RFMIP), Navigation-Grade Integrated Micro Gyroscopes (NGIMG), and Mi- cro Cryogenic Coolers (MCC) programs in the Microsystems Technology Office of DARPA.

Prof. Nguyen received the 1938E Award for Research and Teaching Excellence from the University of Michigan in 1998, an EECS Departmental Achievement Award in 1999, the Ruth and Joel Spira Award for Teaching Excellence in 2000, the University of Michigan's 2001 Henry Russel Award, and the Cady Award from the 2006 IEEE Frequency Control Symposium. Among his publication accolades are the Jack Raper Award from 2005 IEEE International Solid-State Circuits Conference, the 2004 DARPA Tech Best Technical Presentation Award, the Best Invited Paper Award at the 2004 IEEE Custom Integrated Circuits Conference, and together with his students, the Best Student Paper Award in Category 1 at the 2005 Joint IEEE Frequency Control/Precise Time and Timing Interval (PTTI) Symposium, the Best Student Paper Award in the Frequency Control Category at the 2004 IEEE Ultrasonics, Ferroelectrics, and Frequency Control Symposium, and the Roger A. Haken Best Student Paper Awards at the 1998 and 2003 IEEE International Electron Devices Meetings. To date, he has organized and chaired a total of 35 IEEE and DARPA workshops. 